\title{
MSSM $A$-funnel and the galactic center excess: prospects for the LHC and direct detection experiments
}

\author{
Katherine Freese, ${ }^{a, b, c}$ Alejandro López, ${ }^{c}$ Nausheen R. Shah ${ }^{c, d}$ and Bibhushan Shakya ${ }^{c}$ \\ ${ }^{a}$ Nordita (Nordic Institute for Theoretical Physics), \\ KTH Royal Institute of Technology and Stockholm University, \\ Roslagstullsbacken 23, SE-106 91 Stockholm, Sweden \\ ${ }^{b}$ The Oskar Klein Center for Cosmoparticle Physics, AlbaNova University Center, \\ University of Stockholm, \\ 10691 Stockholm, Sweden \\ ${ }^{c}$ Michigan Center for Theoretical Physics, Department of Physics, University of Michigan, \\ Ann Arbor, MI 48109, U.S.A. \\ ${ }^{d}$ Department of Physics and Astronomy, Wayne State University, \\ Detroit, Michigan 48201, U.S.A. \\ E-mail: ktfreese@umich.edu, aolopez@umich.edu, naushah@umich.edu, \\ bshakya@umich.edu
}

ABSTRACT: The pseudoscalar resonance or " $A$-funnel" in the Minimal Supersymmetric Standard Model (MSSM) is a widely studied framework for explaining dark matter that can yield interesting indirect detection and collider signals. The well-known Galactic Center excess (GCE) at $\mathrm{GeV}$ energies in the gamma ray spectrum, consistent with annihilation of a $\lesssim 40 \mathrm{GeV}$ dark matter particle, has more recently been shown to be compatible with significantly heavier masses following reanalysis of the background. In this paper, we explore the LHC and direct detection implications of interpreting the GCE in this extended mass window within the MSSM $A$-funnel framework. We find that compatibility with relic density, signal strength, collider constraints, and Higgs data can be simultaneously achieved with appropriate parameter choices. The compatible regions give very sharp predictions of 200-600 GeV CP-odd/even Higgs bosons at low $\tan \beta$ at the LHC and spin-independent cross sections $\approx 10^{-11} \mathrm{pb}$ at direct detection experiments. Regardless of consistency with the GCE, this study serves as a useful template of the strong correlations between indirect, direct, and LHC signatures of the MSSM $A$-funnel region.

KEYWORDS: Supersymmetry Phenomenology

ARXIV EPRINT: 1509.05076 


\section{Contents}

1 Introduction and motivation 1

2 The MSSM pseudoscalar resonance: dark matter aspects 3

2.1 Dark matter composition 4

2.2 Relic density and signal strength 4

3 Constraints $\quad 6$

$\begin{array}{lll}3.1 & \text { Collider and Higgs sector constraints } & 6\end{array}$

3.2 Vacuum metastability 8

3.3 Direct detection 8

3.4 Indirect detection $\quad 9$

4 Numerical results $\quad 10$

4.1 Fit procedure 11

$\begin{array}{lll}4.2 & \text { Fit results } & 12\end{array}$

$\begin{array}{lll}\text { 4.2.1 Fit to a modified spectrum } & 15\end{array}$

5 Predictions for the LHC and direct detection experiments 16

$\begin{array}{lll}5.1 & \text { LHC prospects } & 16\end{array}$

$\begin{array}{lll}5.2 \text { Direct detection } & 19\end{array}$

$\begin{array}{llr}6 & \text { Summary } & 20\end{array}$

$\begin{array}{ll}\text { A Parameters and vacuum metastability } & 21\end{array}$

\section{Introduction and motivation}

The Galactic Center (GC) of the Milky Way galaxy is the densest dark matter region in our vicinity and has long been earmarked as the most promising target for searches of dark matter (DM) signals. Intriguingly, recent years have seen a persistent and statistically significant excess in the gamma ray spectrum peaking at $2-5 \mathrm{GeV}$ originating from the GC, above what is predicted from known sources and conventional astrophysics [1-12]. The signal was initially reported to be compatible with $\sim 40$ (10) GeV dark matter annihilating into $b \bar{b}(\tau \tau)$, with an annihilation cross section $\langle\sigma v\rangle \sim \mathcal{O}\left(10^{-26}\right) \mathrm{cm}^{3} / \mathrm{s}$. Since this is approximately the annihilation cross section expected of a thermal relic, a dark matter interpretation of this excess presents itself as a very tantalizing possibility. This prospect has been explored by many authors in various contexts (see, for instance refs. [9, 10, 13, 14] and references therein), including the Minimal Supersymmetric Standard Model (MSSM) [15-18]. 
More recently, it has been shown that this excess might be attributable to unresolved point sources [19-21], although a conclusive verdict has not been reached.

Recently, the Fermi-LAT Collaboration has presented an analysis of the region around the GC with four different variants of foreground/background models, finding, for every variant, significant improvements in the agreement with data when an additional component centered at the GC with a peaked profile (NFW, NFW-contracted), i.e. a dark matter-like spectrum, was included in the fits [22, 23] (see also ref. [12] for an attempt at accounting for systematic uncertainties in the background). From a dark matter perspective, a recent study [15] found these additional components for the four choices of background models to be compatible with several annihilation channels $(W W, Z Z, h h, t \bar{t})$ and significantly higher DM masses $(165 \mathrm{GeV}$ for $b \bar{b}, 310 \mathrm{GeV}$ for $t \bar{t}$ ) than previously thought possible. Similar conclusions were also reached in refs. [17] and [18], which reported that a higher mass $(175-200 \mathrm{GeV})$ dark matter annihilating into $t \bar{t}$ could give reasonable fits to the signal.

This relaxation of the allowed range of dark matter masses compatible with the GC excess (GCE) has particularly interesting implications for MSSM dark matter, as it opens up the possibility of explaining the signal with the well-known pseudoscalar resonance or " $A$-funnel" mechanism, where the dark matter relic density is set by resonant $s$-channel annihilation through the pseudoscalar $A$, with $m_{A} \approx 2 m_{\chi}$ ( $\chi$ represents the lightest neutralino, which is the dark matter candidate). The pseudoscalar resonance has been studied in connection with the GCE outside the MSSM in refs. [24-26]; however, realizing the mechanism in the MSSM is of particular interest given that the MSSM remains one of the most familiar and widely studied Beyond the Standard Model (BSM) theories. Previous fits to the GCE with $m_{\chi} \lesssim 50 \mathrm{GeV}$ did not allow for this possibility in the MSSM due to constraints on $m_{A}$ from direct LHC searches [27, 28] (although this constraint can be circumvented in the the Next-to-Minimal Supersymmetric Model (NMSSM), allowing for an NMSSM explanation of the GCE [25, 29]). This incompatibility is lifted if, as discussed in ref. [15], $m_{\chi} \lesssim 165(310) \mathrm{GeV}$ annihilates into $b \bar{b}(t \bar{t})$, allowing for $m_{A}$ large enough to evade collider constraints.

The aim of this paper is to explore whether, given this wider range of allowed masses, the MSSM pseudoscalar resonance can give reasonable fits to the GCE, consistent with stringent constraints from relic density, indirect/direct detection, collider search limits, and Higgs data. Since the mechanism requires a light $(\sim 200-500 \mathrm{GeV})$ pseudoscalar, the SM-like nature of the $125 \mathrm{GeV}$ Higgs boson is particularly constraining as the heavier CPeven Higgs is at the same mass as the pseudoscalar and can mix with the $125 \mathrm{GeV}$ Higgs, resulting in deviations from SM-like properties inconsistent with measurements. For such light, non-decoupled heavier Higgs bosons, the Higgs sector needs to be "aligned" [30-35] to maintain SM-like properties for the $125 \mathrm{GeV}$ mass eigenstate. As we will show in this paper, this can indeed be achieved while simultaneously satisfying all other DM requirements.

A successful realization of neutralino dark matter along with the GCE through the pseudoscalar resonance requires very precise choices of parameters in order to simultaneously achieve resonant annihilation, the Higgs mass, and alignment in the Higgs sector (this is also the reason why extensive scans in the MSSM parameter space [15-18] fail to uncover 
it as a viable explanation of the GCE). It is nevertheless worthwhile to pursue this direction for several reasons. First, the $A$-funnel is one of several "traditional" mechanisms in the MSSM that have been widely studied for a long time, and its compatibility with a possible DM signal is therefore of considerable interest. Second, while most scenarios put forward to explain the GCE could potentially be constrained by stringent spin-independent direct detection limits (indeed, avoiding these limits itself involves some nontrivial fine-tuning of parameters in supersymmetric models [36-38]), the $A$-funnel naturally gives small direct detection cross sections and is automatically safe from these bounds. Most importantly, the framework is eminently predictive, giving very specific predictions for heavy Higgs bosons that will be probed at the $13 \mathrm{TeV}$ LHC and future colliders, as well as direct detection cross sections that may be probed by the next generation of experiments. Independent of these considerations, and independent of the applicability to the GCE, this study serves as a valuable template of the conditions necessary for the existence of a light pseudoscalar in the MSSM together with indirect detection signals of dark matter via the A-funnel.

The outline of the paper is as follows. Section 2 introduces the parameter space relevant for the study and discusses dark matter aspects such as the annihilation cross section and relic density. Section 3 is devoted to a discussion of various constraints from direct detection, indirect detection, collider constraints, Higgs data, and vacuum metastability. Section 4 presents the details of our scans and the best fit regions to the GCE. Predictions for the $13 \mathrm{TeV}$ LHC and future direct detection searches are presented in section 5 . We summarize our results in section 6 . The appendices contains additional details on the MSSM parameters and fits to the GCE.

\section{The MSSM pseudoscalar resonance: dark matter aspects}

In $R$-parity conserving supersymmetric models, the lightest supersymmetric particle (LSP) is stable. If it is also neutral, it can be a dark matter candidate. In the MSSM, the LSP is often assumed to be the lightest of the neutralinos, the neutral superpartners of the gauge bosons and Higgs bosons (Bino, Wino and Higgsinos respectively). The Wino and the Higgsinos tend to annihilate too efficiently to explain the observed dark matter abundance. However, the Bino can yield the correct relic density via various mechanisms, including resonant annihilation via the pseudoscalar, and has long been regarded as the favored dark matter candidate.

We perform our study in the phenomenological MSSM (pMSSM) [39], which is defined in terms of 19 parameters, which are taken to be independent at the weak scale. Of these, our analysis will be entirely determined by the following seven parameters:

- $M_{1}$, the Bino mass parameter. The dark matter is mostly Bino, so this is also approximately the mass of the dark matter candidate $m_{\chi} \approx M_{1}$.

- $\mu$ parameter. This is the Higgsino mass, and controls the Higgsino fraction in the dark matter particle $\chi$. As we will see later, the relic density, signal strength, and direct detection cross section all depend sensitively on this fraction.

- $\tan \beta$, the ratio of the up- and down-type Higgs vacuum expectation values (vevs). 
- $m_{A}$, the heavy Higgs mass. This is the mass of the pseudoscalar that mediates the resonance (hence $m_{A} \approx 2 m_{\chi}$ ) as well as the mass of the heavier scalar, which feeds into Higgs phenomenology and expected direct detection cross-sections.

- $m_{Q_{3}}, m_{u_{3}}$, the left and right handed stop masses, which contribute significantly to the mass of the observed $125 \mathrm{GeV}$ Higgs boson. In this paper we take the stop mass scale $M_{S}^{2} \equiv m_{Q 3}^{2}=m_{u 3}^{2}$.

- $A_{t}$, stop trilinear coupling. This determines the mixing in the stop sector and is again a relevant parameter for the mass of the observed Higgs boson.

All other masses, such as the other gaugino (wino and gluino) and sfermion masses, are assumed to be heavy and decoupled from the analysis.

\subsection{Dark matter composition}

The lightest neutralino in the MSSM is a combination of the Bino, Wino, and neutral Higgsinos:

$$
\chi=N_{11} \tilde{B}+N_{12} \tilde{W}+N_{13} \tilde{H}_{d}+N_{14} \tilde{H}_{u} .
$$

As mentioned above, we are mainly interested in the region of parameters where the lightest neutralino is predominantly a Bino, hence $N_{11} \sim 1, N_{12}=0$, and $N_{13}, N_{14} \ll 1$. In this regime, the Bino mass parameter $M_{1}$ and the neutralino components are approximately [25]

$$
\begin{aligned}
M_{1} & =m_{\chi}+\frac{m_{Z}^{2} s_{W}^{2}\left(\mu s_{2 \beta}+m_{\chi}\right)}{\mu^{2}-m_{\chi}^{2}} \\
\frac{N_{13}}{N_{11}} & =\frac{m_{Z} s_{W} s_{\beta}}{\mu^{2}-m_{\chi}^{2}}\left(\mu+\frac{m_{\chi}}{t_{\beta}}\right) \sim \frac{m_{Z} s_{W}}{\mu} s_{\beta} \\
\frac{N_{14}}{N_{11}} & =-\frac{m_{Z} s_{W} c_{\beta}}{\mu^{2}-m_{\chi}^{2}}\left(\mu+t_{\beta} m_{\chi}\right) \sim-\frac{m_{Z} s_{W}}{\mu} c_{\beta}\left(1+t_{\beta} \frac{m_{\chi}}{\mu}\right), \\
N_{11} & =\left(1+\frac{N_{13}^{2}}{N_{11}^{2}}+\frac{N_{14}^{2}}{N_{11}^{2}}\right)^{-1 / 2} .
\end{aligned}
$$

Here, $s_{\theta}, c_{\theta}$ denote $\sin \theta, \cos \theta$ respectively and $m_{\chi}$ is the dark matter mass.

\subsection{Relic density and signal strength}

Both the relic density and the present day annihilation cross section are driven by the process $\chi \chi \rightarrow f \bar{f}$ with the pseudoscalar $A$ in the $s$-channel (we are interested in the case where the fermion $f$ is either $b$ or $t$ for compatibility with the GCE). When the process occurs close to resonance, it is well-known that the annihilation cross-section in the early universe (which sets the relic density at the time of freeze-out) is substantially different from that at present times (which sets the signal strength fitting the GCE) due to thermal broadening of the resonance during the former stage [40]. Thus, with appropriate parameter choices, one can scale the relic density and the present annihilation cross section independent of each other, thereby achieving better agreement with both measurements; 
this degree of freedom is not afforded in non-resonant scenarios, where these two quantities are strictly related to each other.

To understand this interplay, consider a simplified model describing a Majorana DM particle $\chi$ coupled to a pseudoscalar $A$ through the interaction Lagrangian

$$
-\mathcal{L}_{\text {int }}=i y_{a \chi \chi} A \bar{\chi} \gamma^{5} \chi+i y_{a f f} A \bar{f} \gamma^{5} f .
$$

The entire parameter space of the model is then determined by $m_{A}, m_{\chi}, y_{a \chi \chi}$ and $y_{a f f}$. A crucial parameter in our analysis is the degeneracy parameter

$$
\delta=\left|1-4 m_{\chi}^{2} / m_{A}^{2}\right|,
$$

which characterizes the proximity to the resonant regime. We are interested in scenarios where $\delta \approx 0$.

The resonant annihilation cross-section at a given temperature $T$ is [40]

$$
\langle\sigma v\rangle \simeq \frac{3 e^{-x \delta} x^{3 / 2} \delta^{1 / 2} y_{a \chi \chi}^{2} y_{a f f}^{2} m_{\chi}^{2}}{\sqrt{\pi} m_{A}^{3} \Gamma_{A}},
$$

where $x=m_{\chi} / T$ and $\Gamma_{A}$ is the decay width of $A$,

$$
\Gamma_{A} \simeq \frac{m_{A}}{16 \pi}\left(y_{a \chi \chi}^{2}+6 y_{a f f}^{2}\right)
$$

This gives the relic abundance

$$
\Omega h^{2}=\frac{3.12 \times 10^{-12} m_{A}^{3} \Gamma_{a}}{(\mathrm{GeV})^{2} m_{\chi}^{2} y_{a \chi \chi}^{2} y_{a f f}^{2} \operatorname{Erfc}\left[\sqrt{x_{f} \delta}\right]},
$$

where $x_{f}$ is the value of $x$ at freeze-out. This expression can be rewritten in a more illuminating form as [25]

$$
\Omega h^{2} \sim 0.12\left(\frac{m_{A}^{2}}{4 m_{\chi}^{2}}\right)\left(\frac{m_{A}}{220 \mathrm{GeV}}\right)^{2}\left[\frac{y_{a \chi \chi}^{-2}+(\delta / 6) y_{a f f}^{-2}}{10^{5}}\right]\left(\frac{\operatorname{Erfc}[1.325]}{\operatorname{Erfc}\left[\sqrt{x_{f}} \delta\right]}\right) .
$$

Likewise, the DM annihilation cross-section today is

$$
\left.\sigma v\right|_{v=0} \simeq \frac{3}{2 \pi} \frac{y_{a \chi \chi}^{2} y_{a f f}^{2} m_{\chi}^{2}}{\left(m_{A}^{2}-4 m_{\chi}^{2}\right)^{2}+m_{A}^{2} \Gamma_{A}^{2}} .
$$

Assuming that $m_{A} \sim 2 m_{\chi}$ so that the second term dominates in the denominator, one obtains (for $2 m_{\chi}<m_{a}$ ) [25]

$$
\left.\sigma v\right|_{v=0} \sim 2 \times 10^{-26} \mathrm{~cm}^{3}\left(\frac{4 m_{\chi}^{2}}{m_{A}^{2}}\right)\left(\frac{220 \mathrm{GeV}}{m_{A}}\right)^{2} \frac{10^{-5}}{\left(\frac{y_{a \chi \chi}}{y_{a f f}} \frac{\delta}{6}+\frac{y_{a f f}}{y_{a \chi \chi}}\right)^{2}} .
$$

Comparing eq. (2.8) and eq. (2.10), it is clear that the relic density and the current annihilation cross-section can be independently scaled with judicious choices of $y_{\text {aff }}$ and $y_{a \chi \chi} \sqrt{\delta / 6}$. In terms of the fundamental MSSM parameters, these couplings are given by:

$$
\begin{aligned}
y_{a b b} & =\frac{i m_{b} \tan \beta}{\sqrt{2} v}, \quad y_{a t t}=\frac{i m_{t}}{\sqrt{2} v \tan \beta}, \\
y_{a \chi \chi} & =i g_{1} N_{11}\left(N_{14} \cos \beta-N_{13} \sin \beta\right),
\end{aligned}
$$


where $v=174 \mathrm{GeV}$ and $g_{1}$ is the $\mathrm{SM} \mathrm{U}(1)_{Y}$ gauge coupling. Note from the above that a non-vanishing $y_{a \chi \chi}$ coupling requires a non-vanishing Higgsino component in $\chi$. From the expressions for $N_{11}, N_{13}, N_{14}$ listed previously, we thus see that, for given values of $m_{A}$ and $\tan \beta$, the desired relic density and an annihilation cross-section consistent with the GCE can be obtained simultaneously by appropriately choosing $\mu$ and $\delta$ (equivalently, $m_{\chi}$ ).

\section{Constraints}

As mentioned in section 1, the relevant $A$-funnel parameter space is constrained from several directions. Higgs phenomenology in our set-up is very directly linked to the GCE, hence LHC direct searches as well as the properties of the observed $125 \mathrm{GeV}$ Higgs put stringent constraints on this scenario. Consistency with all collider observables can then create tension with constraints from requiring the stability of the electroweak vacuum. In addition, since the CP-even heavy Higgs $H$ is expected to be approximately degenerate in mass with $A$, contributions to the spin-independent direct detection cross-section from $H$-exchange might be relevant. Finally, there are also several current and future indirect detection experiments that can probe the process of interest in this paper. In this section we detail the current status and future prospects in all of these different directions.

\subsection{Collider and Higgs sector constraints}

In the absence of CP-violation (which we assume in this paper), the physical spectrum of the Higgs sector consists of two CP-even Higgs bosons, $h$ and $H$, one CP-odd state $A$, and a pair of charged Higgs bosons, $H^{ \pm}$. Direct searches for these heavier Higgs bosons at the LHC rule out a significant part of parameter space. ATLAS and CMS direct searches for charged Higgs bosons $[27,28]$ rule out $m_{H+} \leq 160 \mathrm{GeV}$ (recall that $m_{H+}^{2}=m_{A}^{2}+m_{W}^{2}$ at tree level). Likewise, there exist strong limits from searches for $A / H \rightarrow \tau \tau$ [41], which provide the strongest limits, although these depend on $\tan \beta$ and can be evaded for small values of $\tan \beta .^{1}$

Beyond these direct constraints, a small $m_{A}$ is still in tension with Higgs data, as a light CP-even Higgs $\left(m_{H} \approx m_{A}\right.$ in the MSSM) tends to mix with the $125 \mathrm{GeV}$ state and cause deviations from SM-like properties. This is a particularly strong constraint in our framework and dictates what values our parameters can take, hence we will now study this constraint in some detail.

The MSSM Higgs sector consists of two doublets, $H_{u}$ and $H_{d}$; the former couples to all the up-type fermions and the latter to the down-type fermions and charged leptons. The neutral components acquire vacuum expectation values $v_{u}$ and $v_{d}$ with $\tan \beta=t_{\beta}=v_{u} / v_{d}$ and $v^{2}=\sqrt{v_{u}^{2}+v_{d}^{2}}=174 \mathrm{GeV}$. One can define a "Higgs-basis", where a single field acquires

\footnotetext{
${ }^{1}$ Light $m_{A} / m_{H}$ and heavily mixed stops (as usually needed for a $125 \mathrm{GeV}$ Higgs in the MSSM) can also give large contributions to various flavor observables, for example $B_{s} \rightarrow \mu^{+} \mu^{-}$and $B \rightarrow X_{s} \gamma$. However, in this work we will mainly be interested in moderate to small value of $\tan \beta$, hence there is no large enhancement of these effects. Moreover, the size of these contributions are heavily dependent on the signs of various contributions (see e.g. ref. [42]), and consistency with all measured values could be obtained by tuning such cancellations.
} 
all the vev:

$$
\begin{aligned}
H_{S M} & =s_{\beta} H_{u}+c_{\beta} H_{d}, \\
H_{N S M} & =-c_{\beta} H_{u}+s_{\beta} H_{d},
\end{aligned}
$$

where $s_{\beta} \equiv \sin \beta, c_{\beta} \equiv \cos \beta,\left\langle H_{S M}\right\rangle=v$, and $\left\langle H_{N S M}\right\rangle=0$. The couplings of these states to the SM fields are:

$$
\begin{aligned}
g_{H_{S M}}^{d d / u u / V V} & =g^{S M} \\
g_{H_{N S M}}^{d d}=g^{S M} t_{\beta}, \quad g_{H_{N S M}}^{u u} & =-g^{S M} / t_{\beta} \quad g_{H_{N S M} V}^{V V}=0,
\end{aligned}
$$

where $V V, u u, d d$ refer to all vector, up-type and down-type states respectively, and $g^{S M}$ refers to the SM value of these couplings. Note that there is no coupling between the $H_{N S M}^{0} / A$ states and the $H_{S M}^{0}$ or between the gauge bosons and $H_{N S M}^{0}$.

The mass eigenstates, $h$ and $H$, can be written as mixtures of the Higgs basis fields,

$$
\begin{aligned}
h & =\kappa_{S M}^{h} H_{S M}+\kappa_{N S M}^{h} H_{N S M}, \\
H & =\kappa_{S M}^{H} H_{S M}+\kappa_{N S M}^{H} H_{N S M},
\end{aligned}
$$

where $\kappa_{N S M}^{h}=-\kappa_{S M}^{H}=c_{\alpha-\beta} \equiv \cos (\alpha-\beta)$ and $\kappa_{S M}^{h}=\kappa_{N S M}^{H}=s_{\alpha-\beta} \equiv \sin (\alpha-\beta)$, and $\alpha$ is the angle of rotation from the $\left(H_{u}, H_{d}\right)$ basis to the mass eigenstates. We want to identify the lightest $\mathrm{CP}$-even mass eigenstate, $h$, with the recently observed $125 \mathrm{GeV}$ scalar; given that all measurements suggest that its properties are SM-like, we also want to identify it as the SM-like field in the Higgs basis. That is, we require

$$
h_{125}=h \approx H_{S M} .
$$

This requirement of vanishing mixing between the $H_{N S M}$ state and the $125 \mathrm{GeV}$ Higgs, corresponding to $\kappa_{N S M}^{h} \approx 0$, can be rewritten in terms of the fundamental parameters as $[30,31]$

$$
t_{\beta} c_{\beta-\alpha} \simeq \frac{-1}{m_{H}^{2}-m_{h}^{2}}\left[m_{h}^{2}+m_{Z}^{2}+\frac{3 m_{t}^{4} X_{t}\left(Y_{t}-X_{t}\right)}{4 \pi^{2} v^{2} M_{S}^{2}}\left(1-\frac{X_{t}^{2}}{6 M_{S}^{2}}\right)\right] \simeq 0,
$$

where $M_{S}$ is the geometric mean of the stop masses and

$$
X_{t} \equiv A_{t}-\mu / t_{\beta}, \quad Y_{t} \equiv A_{t}+\mu t_{\beta} .
$$

Note that when the second Higgs becomes heavy $\left(m_{H} \gg m_{h}\right)$, this relation is automatically satisfied; this is the familiar decoupling effect. Otherwise, one requires alignment without decoupling [30, 31], brought about by an accidental cancellation in the fundamental parameters of the theory so as to satisfy eq. (3.6). For small $t_{\beta}$ and $M_{S} \sim \mathcal{O}(1) \mathrm{TeV}$, large values of $A_{t} / M_{S}$ are required to obtained an experimentally consistent Higgs mass whereas large values of $\left(\mu A_{t}\right) / M_{S}^{2}$ lead to close to alignment conditions [30, 31].

The CMS and ATLAS collaborations present both the precision measurements of the $125 \mathrm{GeV}$ Higgs and the searches for $H \rightarrow W W / Z Z$ as ratios to the expectations from a 
SM Higgs of the same mass. The predicted rate at the LHC for the decay of the mass eigenstate $i=\{h, H\}$ into some final state $X X$ as a ratio to the SM value is given by

$$
\mathcal{R}_{X X}^{i}=\left(\sigma^{i} / S M\right) \times\left(B R_{X X}^{i} / S M\right)
$$

where $S M$ in the denominators denote the corresponding values for a SM-like Higgs of the same mass. For a $125 \mathrm{GeV}$ SM-like Higgs, the dominant decay mode is into a pair of $b$-quarks $(\sim 60 \%)$, followed by $W W$; hence the total width is dominated by the width into $b$ quarks. The largest deviation from mixing effects is expected in the precision measurements of $h \rightarrow W W$. This number is reported to be $\mathcal{R}_{W W}^{h}=1.16_{-0.21}^{+0.24}$ by ATLAS [43] and $\mathcal{R}_{W W}^{h}=0.83 \pm 0.21$ by CMS [44]. In our analysis we will take a conservative approach of assuming that observational consistency is obtained (that is, the Higgs sector is sufficiently aligned) for $\mathcal{R}_{W W}^{h}$ between $0.7-1.3$. This range will narrow with additional data, and measurements at the level of $10 \%$ are expected at the high luminosity LHC [45, 46].

\subsection{Vacuum metastability}

Another important constraint on these parameters comes from vacuum metastability. Large values of the soft stop trilinear coupling $A_{t}$, required for the Higgs mass and alignment (discussion above), can result in the appearance of charge- and color-breaking minima in the scalar potential of the MSSM. The condition for either these minima to be energetically unfavorable or the tunneling to these minima to have lifetimes longer than the age of the Universe leads to the approximate bound [47]

$$
A_{t}^{2} \lesssim\left(3.4-0.5 \frac{|1-r|}{1+r}\right) m_{T}^{2}+60 m_{2}^{2}
$$

where $m_{T}^{2}=m_{Q_{3}}^{2}+m_{u_{3}}^{2}, m_{2}^{2}=m_{H_{u}}^{2}+\mu^{2}$, and $r=m_{u_{3}}^{2} / m_{Q_{3}}^{2}$. In our analysis we assume $m_{Q 3}^{2}=m_{u 3}^{2} \equiv M_{S}^{2}$, so that $r=1$. Minimization conditions of the Higgs potential give $m_{2}^{2}=m_{A}^{2} \cos ^{2} \beta+0.5 m_{Z}^{2} \cos (2 \beta)$, hence the condition for vacuum metastability can be written as

$$
A_{t}^{2} \lesssim 6.8 M_{S}^{2}+60 m_{A}^{2} \cos ^{2} \beta+30 m_{Z}^{2} \cos (2 \beta) .
$$

It is worth keeping in mind that this is only an approximate bound and depends on several assumptions (see ref. [47] for details). However, consistency with the above provides a rough guide for the feasibility of the parameter region under investigation.

\subsection{Direct detection}

Direct detection possibilities focusing on the $A$-funnel in the MSSM have been studied in refs. [48-50]. The pseudoscalar $A$ does not mediate spin-independent WIMP-nucleon scattering. Instead this cross section $\sigma_{S I}$ comes from light and heavy CP-even Higgs boson exchanges in the $t$-channel, facilitated by the Bino-Higgsino mixture of the LSP necessary to obtain the correct relic density. There are also contributions from tree level squark exchange in the $s$-channel and from gluon loops [51, 52], but these are negligible when the sfermions are heavy. The cross section then depends only on $M_{1}, m_{A}, \tan \beta$ and $\mu$. 
For given values of $m_{A}$ and $\tan \beta$, requiring the correct relic density and GCE leaves no free parameters, thereby fixing the direct detection cross section. This cross section in our region of interest can be written as approximately [25]

$$
\sigma_{S I} \simeq \frac{m_{Z}^{2} s_{W}^{2} m_{p}^{2} m_{r}^{2}}{\pi v^{4}} N_{11}^{4}\left[\frac{\left(\frac{F_{u}}{t_{\beta}}-F_{d} t_{\beta}\right)}{m_{A}^{2}}\left(\frac{N_{14}}{N_{11}} c_{\beta}+\frac{N_{13}}{N_{11}} s_{\beta}\right)-\frac{\left(F_{d}+F_{u}\right)}{m_{h}^{2}}\left(\frac{N_{13}}{N_{11}} c_{\beta}-\frac{N_{14}}{N_{11}} s_{\beta}\right)\right]^{2},
$$

where $F_{u} \sim 0.15, F_{d} \sim 0.13$ (the up and down type quark content respectively of the nucleon, proton or neutron), $t_{\beta}=\tan \beta, m_{N}$ is the mass of the nucleon, and $m_{r}=\frac{m_{N} m_{\chi}}{m_{N}+m_{\chi}}$ is the reduced mass. For the correct dark matter relic density obtained via the $A$-funnel, this cross section is generally around $10^{-11} \mathrm{pb}$ [48-50,53], well below existing bounds from XENON100 [54] and LUX [55], which currently rule out $\sigma_{S I} \gtrsim 5 \times 10^{-10} \mathrm{pb}$. Note that while the annihilation processes that determine the relic density as well as indirect detection signals are $s$-channel and therefore enhanced by the resonance, the direct detection cross-section is mediated by $t$-channel processes and does not receive this enhancement. Such small direct detection cross sections are therefore a generic feature of this region of parameter space. Crucially, this cross section still lies above the neutrino background and is therefore within reach of future detectors, although detection will still be challenging.

As is well-known, an exception to this generic feature can occur for negative values of the $\mu$ parameter due to destructive interference between the light and heavy Higgs exchange contributions, giving cross sections several orders of magnitude below the neutrino background cross section $[48,50]$. Such blind spots can in general occur at any dark matter mass, but their appearance in the $A$-funnel framework is more strongly constrained as we also need $m_{H} \sim m_{A} \sim 2 m_{\chi}$. Approximating the up- and down-type quark content in the nucleus as roughly equal, this cancellation condition in the $A$-funnel region can be formulated as approximately [48]

$$
m_{A} \sim\left(-2 \mu m_{h}^{2} \tan \beta\right)^{1 / 3} .
$$

With $\mathrm{TeV}$ scale values of $\mu$ necessitated by relic density constraints and $\mathcal{O}(1)$ values of $\tan \beta$ required by collider constraints (see section 3.1), eq. (3.12) implies that the cancellation can only occur for large $m_{A} \gtrsim 650 \mathrm{GeV}$, beyond the mass range of interest from the point of view of the $\mathrm{GeV}$ excess. Hence all parameter combinations of interest should predict a small but tractable $\left(\sim 10^{-11} \mathrm{pb}\right)$ direct detection cross section (we will see in the subsequent sections that this in indeed realized, see figure 7 ).

\subsection{Indirect detection}

Currently the strongest bounds on the annihilation cross section are given by the Fermi/LAT analysis of 6 years of data on 15 known dwarf galaxies [56]. For $100-300 \mathrm{GeV}$ dark matter, which is our region of interest, this analysis constrains the annihilation crosssection to be less than $\sim$ a few $\times 10^{-26} \mathrm{~cm}^{3} / \mathrm{s}$. The cross section required to explain the GCE is also in this region over this mass range (see [15]), hence the dwarf constraints are 
in some tension with a DM interpretation of the GCE. However, the large uncertainties in the dark matter distribution ( $J$-factor) in these dwarf galaxies leave room for compatibility (see figure 8 in ref. [56]). For instance, the 95\% C.L. annihilation cross-section exclusion limit for a $100 \mathrm{GeV}$ WIMP annihilating to $b \bar{b}$ is $2.2 \times 10^{-26} \mathrm{~cm}^{3} / \mathrm{s}$ and has a $1 \sigma$ error interval of $\left[9.0 \times 10^{-27}, 5.6 \times 10^{-26}\right] \mathrm{cm}^{3} / \mathrm{s}$, which is compatible with the cross section interval $\left[3.1 \times 10^{-27}, 8.8 \times 10^{-26}\right] \mathrm{cm}^{3}$ needed to fit to the GCE at this mass. A signal was reportedly seen in the new dwarf galaxy candidate Reticulum II [57], found in the first year DES data [58], consistent with a dark matter of mass $\sim 40-200 \mathrm{GeV}$ annihilating into $b \bar{b}$ with a cross section $\langle\sigma v\rangle \sim 10^{-26} \mathrm{~cm}^{3} / \mathrm{s}$, although this was later found to be inconsistent with the new PASS 8 diffuse emission model used to analyze Reticulum II [59]. Bounds similar to those from the Fermi dwarf observations are also found by the Planck satellite from CMB measurements [60].

Likewise, since DM of interest in this paper annihilates primarily through hadronic channels $(b \bar{b}$ and $t \bar{t})$, this is expected to generate a significant flux of antiprotons. There already exists some tension between models that explain the GCE and derived constraints from antiproton bounds on dark matter annihilation [61-63]. However, calculation of the antiproton flux suffers from significant uncertainties related to the propagation model in the galaxy (see [63-66] and references therein), and the GCE can be made compatible with the measured antiproton flux for conservative choices of propagation model parameters.

Bounds on the dark matter annihilating cross-section into quarks are also obtained by neutrino experiments like IceCube. The most current results from the IceCube-79 experiment exclude $\langle\sigma v\rangle \geq 2 \times 10^{-22} \mathrm{~cm}^{3} / \mathrm{s}$ into $b \bar{b}$ at $90 \%$ confidence level [67]. This lower limit is $\sim 10^{4}$ larger than the cross-section required for the GCE [15] and thus irrelevant.

Therefore, no indirect detection results robustly rule out a DM interpretation of the GCE at present, although future measurements, particularly from Fermi-LAT observation of dwarfs, AMS-02 antiproton results, and the CMB could have interesting implications.

\section{Numerical results}

Building on the parameter space and constraints described in the previous sections, we present the fits to the GCE excess in this section. We used the following tools for our numerical analysis: the neutralino relic abundance and annihilation crosssection was calculated with Micromegas-4.1.7 [68], the MSSM particle spectra were computed using SuSpect-2.41 [69], and the Higgs phenomenology was obtained with FeynHiggs-2.11.0 [70-74].

For the gamma ray spectrum corresponding to the signal, we follow the approach employed in ref. [15] and consider two of the four spectra presented in figure 13 of ref. [23], ${ }^{2}$ which were derived by fitting the excess over various choices of background as exponentially cut off power laws (see ref. [15, 22] for further details). The four spectra are referred to as spectra (a)-(d) in ref. [15], and just as they do, we pick spectra (b) and (d) for our

\footnotetext{
${ }^{2}$ The first version of our paper used the spectra presented in ref. [22], and ref. [23] is the corresponding publication that recently appeared; we have chosen the spectra from ref. [23] that correspond most closely to the spectra we used in the first version.
} 
analysis; spectrum (a) is very similar to what has been studied for light $\left(m_{\chi} \lesssim 40 \mathrm{GeV}\right.$ ) DM in previous papers and not amenable to the MSSM, whereas spectrum (c) is very similar to spectrum (d) and does not yield any new insight.

Spectrum (b) corresponds to a fit with OB stars as cosmic ray (CR) sources and a tuned index for pion production within the solar circle (see [22, 23]); the analysis in ref. [15] found it to be well fit by $75-95 \mathrm{GeV} \mathrm{DM}$ annihilating into $b \bar{b}$ or $\lesssim 200 \mathrm{GeV} \mathrm{DM}$ annihilating into $t \bar{t}$. Annihilation into gauge or Higgs bosons were also found to give good fits, but these are irrelevant for our analysis since they are always subdominant channels in the MSSM pseudoscalar resonance scenario. Note that spectrum (b) is also in agreement with other studies performed in refs. [17] and [18], which also found that $175-200 \mathrm{GeV} \mathrm{DM}$ annihilating into $t \bar{t}$ could be compatible with the GCE. Likewise, spectrum (d) corresponds to a fit with $\mathrm{OB}$ stars as cosmic ray (CR) sources but with only the intensity of pion production tuned (using pulsars instead of OB stars gives a very similar spectrum); ref. [15] found it to correspond to higher mass DM, with $130-165 \mathrm{GeV}$ DM annihilating into $b \bar{b}$ or $250-310 \mathrm{GeV}$ DM annihilating into $t \bar{t}$ giving good fits.

In this section, we will perform fits to the two spectra (b) and (d) with the idea of gaining intuition about the range of possibilities that the GCE allows for the MSSM pseudoscalar resonance. We note that the continuous region spanning spectra (b) and (d) could also plausibly explain the GCE for some reasonable background, but do not pursue this direction any further.

\subsection{Fit procedure}

The astrophysical information regarding the distribution of dark matter is encoded in the $J$-factor

$$
J=\frac{1}{\Delta \Omega} \int_{\Delta \Omega} \int_{\text {l.o.s. }} \rho(r)^{2} d s d \Omega=\mathcal{J} \times \bar{J}_{\text {can. }},
$$

where $\Delta \Omega$ is the region of interest (ROI), l.o.s. stands for line of sight, and $\rho$ is the dark matter density. $\bar{J}_{\text {can. }}=2.0 \times 10^{23} \mathrm{GeV}^{2} / \mathrm{cm}^{5}$ is the canonical value of the $J$-factor obtained from evaluating the integral with an NFW profile. Following the analyses in ref. [15], we parametrize the uncertainty in the dark matter density profile with the factor $\mathcal{J}$, which is allowed to vary between $[0.14,4]$.

The gamma-ray spectrum is computed for the following MSSM parameters:

- The pseudoscalar mass is allowed to vary over $200 \mathrm{GeV} \leq m_{A} \leq 700 \mathrm{GeV}$. Below $200 \mathrm{GeV}$, we find that the Higgs sector cannot be sufficiently aligned while remaining consistent with bounds from $H / A \rightarrow \tau^{+} \tau^{-}$from the $8 \mathrm{TeV}$ LHC run. We terminate the scan at $700 \mathrm{GeV}$ since good fits to the GCE (either spectrum (b) or (d)) are not expected for $m_{\chi} \geq 310 \mathrm{GeV}$.

- $\tan \beta$ is scanned over the range $4 \leq \tan \beta \leq 10$. Below $\tan \beta=4$, extremely heavy (multi-TeV) stop masses are required to reproduce the Higgs mass, and large log resummations become important. Above $\tan \beta \sim 10, m_{A} \lesssim 350 \mathrm{GeV}$ is inconsistent with the LHC $H / A \rightarrow \tau^{+} \tau^{-}$bound. Masses heavier than this do not give good fits to the GCE since $m_{A} \gtrsim 310 \mathrm{GeV}\left(m_{\chi} \gtrsim 165 \mathrm{GeV}\right)$ requires annihilation primarily into 
$t \bar{t}$, but for large values of $\tan \beta$ the leading annihilation channel for the pseudoscalar is into $b \bar{b}$.

- For given values of $m_{A}$ and $\tan \beta$, we next scan over $\delta$ (equivalently, $m_{\chi}$ as shown in eq. (2.4)) and $\mu$ for points such that

- the relic density constraint is satisfied: the neutralino makes up all of dark matter $\left(0.08 \leq \Omega h^{2} \leq 0.16\right)$; and

- the annihilation cross section $\langle\sigma v\rangle$ is within the $2 \sigma$ best-fit annihilation crosssection contours from ref. [15].

We scan over $\delta \in[0,0.1]$ in order to stay close to resonance, and over $\mu \in[0.7,10] \mathrm{TeV}$ in order to obtain a mostly bino DM.

- Next, we scan over the stop masses $\left(M_{S}=m_{Q_{3}}=m_{u_{3}}\right) \in[0.7,12.7] \mathrm{TeV}$ and the stop trilinear coupling $A_{t} \in[5,25] \mathrm{TeV}$ for points satisfying

$-122 \leq m_{h} \leq 128 \mathrm{GeV}$; and

- alignment in the Higgs sector.

We take the branching ratio to $W W$ normalized to the SM value $\mathcal{R}_{W W}^{h}$ to be a measure of alignment and select (for each $m_{A}, \tan \beta, \mu, m_{\chi}$ combination) the combination of $M_{S}$ and $A_{t}$ that gives $\mathcal{R}_{W W}^{h}$ closest to 1 while maintaining $122 \leq m_{h} \leq 128 \mathrm{GeV}$.

- All other MSSM input parameters (gaugino/wino masses, trilinear couplings, slepton/squark masses) are set to $5 \mathrm{TeV}$ so that they decouple from this analysis.

The goodness of fit is obtained by performing a $\chi^{2}$ analysis between the gamma-ray spectrum obtained from Micromegas and the GCE (Fermi spectra (b) and (d)). For a given MSSM point, the $\chi^{2}$ is calculated as:

$$
\chi^{2}=\sum_{k} \frac{\left(E_{k}^{2} \frac{d N}{d E_{k}}\left(m_{\chi}, \overline{\mathcal{J}}\langle\sigma v\rangle\right)-E_{k}^{2}\left(\frac{d N}{d E_{k}}\right)_{\mathrm{obs}}\right)^{2}}{\sigma_{k}^{2}},
$$

where the subscript $k$ runs over the 20 energy bins of the Fermi/LAT measurement [22], $d N / d E$ is the gamma-ray spectrum obtained from Micromegas, the subscript obs denotes the spectrum consistent with the Fermi excess (i.e. spectrum (b) or (d)), $\sigma_{k}$ denotes the statistical uncertainty [15], and $\overline{\mathcal{J}}$ is the value of $\mathcal{J} \in[0.14,4]$ that minimizes the $\chi^{2}$ value. The $\chi^{2}$ analysis includes statistical errors, but neglects possible systematic errors from modeling backgrounds near the Galactic Center.

\subsection{Fit results}

The fits resulting from the above procedure are presented in figure 1 as contours of $\chi^{2}$ in the $m_{A}$-tan $\beta$ plane for Fermi spectrum (b) and (d). The pink crosses in each panel denote the points with the best fit to the corresponding spectrum; the gamma-ray spectra of these best fit points are presented in figure 2 along with the MSSM parameters. ${ }^{3}$ In

\footnotetext{
${ }^{3}$ It is worth keeping in mind that the absolute value of $\chi^{2}$ does not have a proper statistical significance without a full analysis of all uncertainties in the signal and theory prediction.
} 

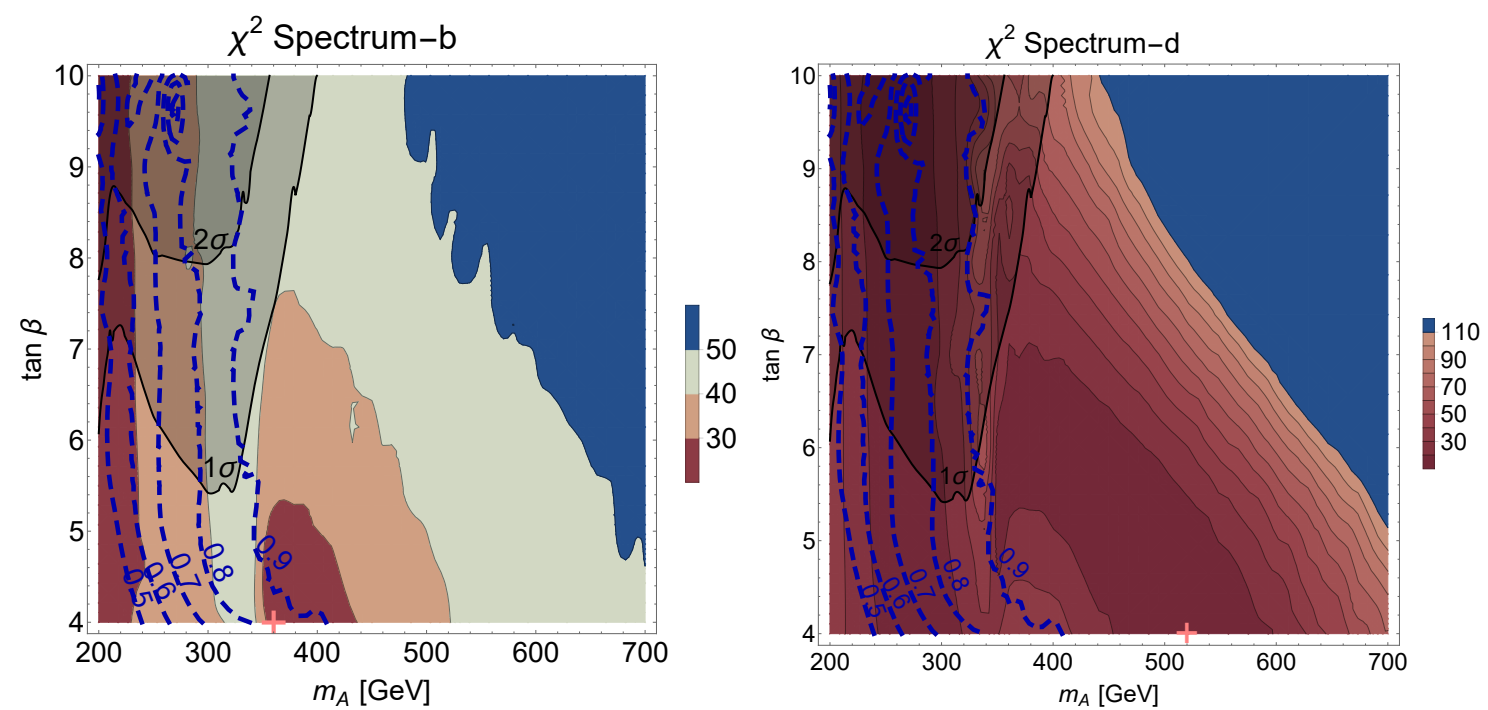

Figure 1. Contours of $\chi^{2}$ in the $m_{A}$-tan $\beta$ plane from fitting the gamma-ray spectrum from the MSSM pseudoscalar resonance to Fermi spectrum (b) (left panel) and spectrum (d) (right panel), corresponding to "OB stars index scaled" and "OB stars intensity scaled" spectra from figure 13 of ref. [23] (see ref. [15, 22, 23] for further details). Red (blue) contour regions denote the best (worst) fits. The $\chi^{2}$ contours are plotted in intervals of 10 in the range $20 \leq \chi^{2} \leq 110$. The pink crosses denote the lowest $\chi^{2}$ value in the scan and hence represent the best fit points. Solid black lines mark the 1- $\sigma$ and 2- $\sigma$ exclusion limits (shaded region above the solid black lines excluded) from the negative search results for $H / A \rightarrow \tau^{+} \tau^{-}$at the $8 \mathrm{TeV}$ LHC run. Dashed blue lines denote contours of the ratio $\mathcal{R}_{W W}^{h}$; current Higgs data from the $8 \mathrm{TeV}$ LHC favors $0.7 \lesssim \mathcal{R}_{W W}^{h} \lesssim 1.3$ (see text for details).

figure 1 we also include, in solid black lines, the 1- $\sigma$ and $2-\sigma$ bounds from $A / H \rightarrow \tau^{+} \tau^{-}$ searches at the $8 \mathrm{TeV}$ LHC [41]; points that lie above these curves in the shaded region are inconsistent with these bounds. These $\tau \tau$ searches, however, lose sensitivity at low $\tan \beta$, hence light pseudoscalars can mediate DM annihilations capable of explaining the GCE in this region. The dashed blue lines correspond to contours of $\mathcal{R}_{W W}^{h}$ as defined in eq. (3.8). $\mathcal{R}_{W W}^{h}=1$ represents a completely SM-like Higgs, and any mixing with the nonSM Higgs causes deviations. Current Higgs data from the LHC allow for $0.7 \lesssim \mathcal{R}_{W W}^{h} \lesssim 1.3$, as discussed in section 3.1. This leads to the requirement of large $\mu$ and hence small couplings [cf. eqs. (2.2) and (2.12)] between $A$ and $\chi$. This generically requires close to resonance conditions $2 m_{\chi} \approx m_{A}$ for consistency with both the GCE and relic density.

We found that the $\chi^{2}$ value did not change significantly between distinct values of $\left(\mu, \delta, A_{t}\right.$, and $M_{S}$ ) for the same $m_{A}, \tan \beta$. This is expected, since the fit quality is driven by the shape of the spectrum, which is controlled mainly by $\tan \beta$ via the branching ratios, and the position of the peak, which is controlled by $m_{A}\left(\approx 2 m_{\chi}\right)$. Although the fit should also depend on the signal strength, which is controlled by $\mu$ and $\delta$ via the annihilation cross section and relic density, the freedom in choosing $\mathcal{J} \in[0.14,4]$, which essentially rescales the signal strength, smears out this dependence. In our region of interest, we find that $\delta \lesssim 0.04$ while $M_{S}, A_{t}$, and $\mu$ all take multi-TeV values; we present contour plots of these 

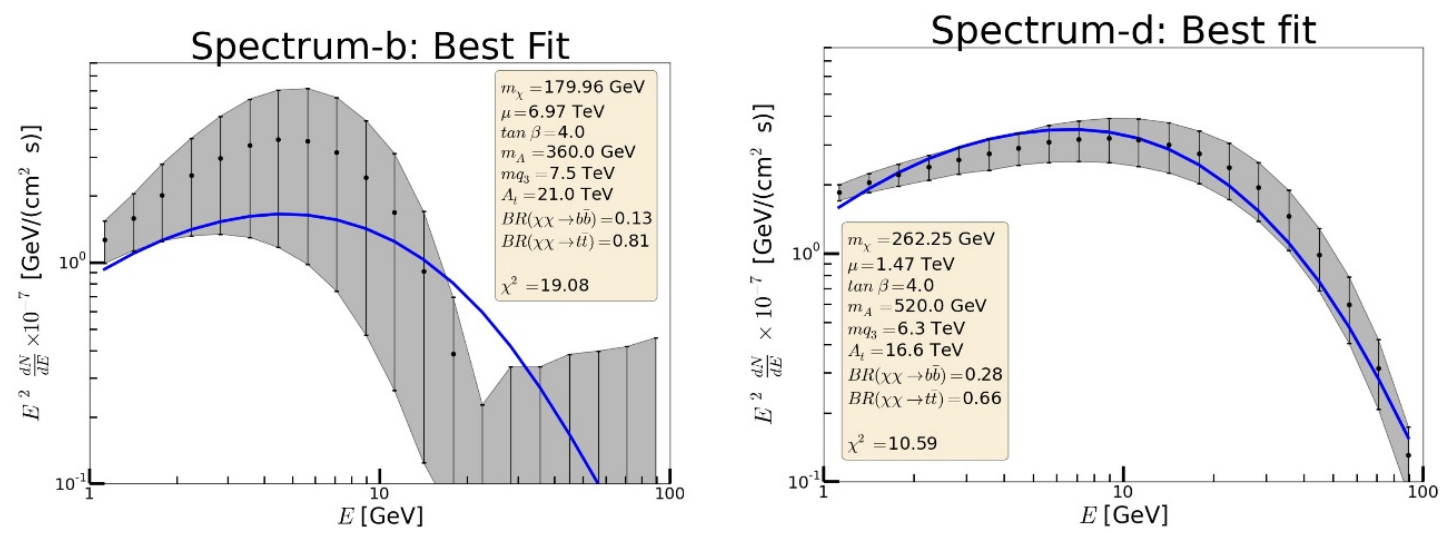

Figure 2. Gamma-ray spectra for the best-fit points corresponding to the pink crosses in figure 1. The gamma-ray spectra from Micromegas (blue line) for the best fit points are superimposed on Fermi spectrum (b) and spectrum (d) (black points) on the left and right panels respectively. The gray band denotes statistical uncertainties (from [23]). Numerical values of the corresponding MSSM parameters and the leading DM annihilation channels are also listed. The value of the higgs mass, relic density, annihilation cross-section and spin-independent scattering cross-section $\left(m_{h}\right.$, $\left.\Omega h^{2},\langle\sigma v\rangle, \sigma_{S I p}\right)$ for the best fit point of spectrum-b/d are $\left(126 \mathrm{GeV}, 0.082,3.849 \times 10^{-26} \mathrm{~cm}^{3} / \mathrm{s}\right.$, $\left.1.689 \times 10^{-12} \mathrm{pb}\right) /\left(127 \mathrm{GeV}, 0.11,3.56 \times 10^{-26} \mathrm{~cm}^{3} / \mathrm{s}, 4.392 \times 10^{-11} \mathrm{pb}\right)$.

parameters in figure 8 in appendix A. The condition for vacuum metastability, eq. (3.10), is also found to be satisfied in most parts of the parameter space allowed by the $8 \mathrm{TeV}$ LHC $A / H \rightarrow \tau^{+} \tau^{-}$bounds (see figure 9 in appendix A).

From the left panel of figure 1, the best fit regions to Fermi spectrum (b) appear to be separated into two distinct islands. The $m_{A} \lesssim 250 \mathrm{GeV}$ region has relatively low $\chi^{2}$ for all values of $\tan \beta$. In this region, annihilation into top quark pairs is kinematically forbidden, so the dominant annihilation channels is $b \bar{b}$ for all values of $\tan \beta$. Recall that an approximately $100 \mathrm{GeV}$ DM particle annihilating into $b \bar{b}$ can fit the GCE [15]; this region reflects this behavior. However, we see that this region is incompatible with the $8 \mathrm{TeV}$ LHC $A / H \rightarrow \tau^{+} \tau^{-}$bounds and/or the Higgs data (that is, $\mathcal{R}_{W W}^{h} \lesssim 0.7$ in this region, signaling that the heavier CP-even scalar is so light that alignment does not work well). A second island opens up at $350 \mathrm{GeV} \lesssim m_{A} \lesssim 450 \mathrm{GeV}$, when annihilation into $t \bar{t}$ becomes kinematically feasible, and $\tan \beta \lesssim 6$. This is consistent with ref. [15] finding a $\sim 200 \mathrm{GeV}$ $\mathrm{DM}$ annihilating into $t \bar{t}$ providing a good fit to spectrum (b). Note that the best fit point occurs at the lowest allowed value of $\tan \beta(=4)$ in our scan, where the coupling of $A$ to top quarks is the largest. The fit deteriorates as $\tan \beta$ gets larger, as the branching ratio into $b \bar{b}$ gets larger due to the $\tan \beta$ enhancement of the $A b b$ coupling. This region is also compatible with Higgs data as $\mathcal{R}_{W W}^{h} \gtrsim 0.7$, and safe from the current $A / H \rightarrow \tau^{+} \tau^{-}$ bounds. Beyond this island, the fit deteriorates rapidly as $m_{A}$ and/or $\tan \beta$ are increased.

Similar patterns are observed for the fit to spectrum (d). A small region of good fit exists at $m_{A} \sim 300 \mathrm{GeV}$ and low $\tan \beta$, safe from the $A / H \rightarrow \tau^{+} \tau^{-}$bounds and borderline compatible with Higgs data. Again, DM in this region annihilates dominantly to $b \bar{b}$ since $t \bar{t}$ is kinematically forbidden, and this observation is compatible with ref. [15], where DM 


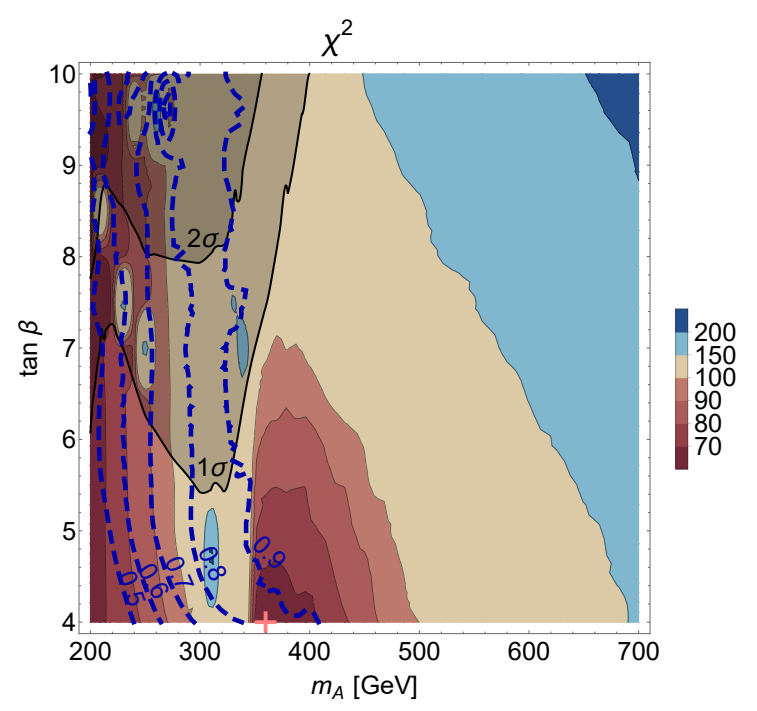

Figure 3. Contours of $\chi^{2}$ in the $m_{A}$-tan $\beta$ plane from fitting the gamma-ray spectrum from the MSSM pseudoscalar resonance to the "pulsars index scaled" spectrum from figure 18 of ref. [23]. Red (blue) contour regions denote the best (worst) fits. Black and blue contours are as in figure 1.

with mass $130-165 \mathrm{GeV}$ annihilating into $b \bar{b}$ was found to give good fits to the spectrum. A second region with better fits is again observed for larger $m_{A}$ once decay into $t \bar{t}$ opens up. This regions roughly spans $450 \mathrm{GeV} \lesssim m_{A} \lesssim 600 \mathrm{GeV}$ and $\tan \beta \lesssim 8$, and appears to correspond to the $250-310 \mathrm{GeV} \mathrm{DM}$ annihilating into $t \bar{t}$ region reported in ref. [15] as a good fit to spectrum (d). Similarly to spectrum (b), the best fit occurs for small values of $\tan \beta: \tan \beta \sim 4$.0. This suggests that a DM candidate that annihilates significantly into $t \bar{t}$ with $B R(\chi \chi \rightarrow t \bar{t})=0.66$ at the best fit point) provides the best fit to spectrum (d). This can be confirmed by comparing the shape of the spectrum in figure 2, right panel, which fits the shape of Fermi spectrum (d) quite well. Finally, the fit deteriorates for larger $m_{A}$ and $\tan \beta$ values and we do not expect any good fits beyond the region shown in the plot.

\subsubsection{Fit to a modified spectrum}

So far, we performed fits to spectra (b) and (d) as defined in ref. [15], corresponding to the "OB stars index scaled" and "OB stars intensity scaled" spectra from figure 13 of ref. [23], which were obtained by modeling the excess with an NFW profile with a single power law with an exponential cutoff. This mimics what is expected of a dark matter source, and serves the purpose of demonstrating how the preferred theory parameter space changes for two different choices of interstellar emission models of the background (matching the philosophy in ref. [15]). However, ref. [23] also finds significantly better fits to the excess if more freedom is allowed in the fit - in particular, if the spectrum of the NFW profile is modeled with a power-law that is allowed to vary per energy band over the $1-100 \mathrm{GeV}$ range; the resulting spectra for various choices of interstellar emission models are presented in figure 18 of ref. [23]. In order to study how the MSSM fit is affected if the latter is used, we performed a similar fit (as described above) to the "pulsars index-scaled" spectrum from figure 18 of ref. [23]; the result is shown in figure 3. We find that the overall fit 
quality worsens due to the tail of the spectrum, but the best fit regions in the MSSM parameter space still closely match those from the fit for spectrum (b) (see figure 1 (left)); consequently, the theoretical implications from fitting to spectrum (b) (discussed below) will also apply in this case.

\section{Predictions for the $\mathrm{LHC}$ and direct detection experiments}

\section{$5.1 \quad$ LHC prospects}

There are several projections for the $14 \mathrm{TeV}$ LHC provided by the CMS and ATLAS collaborations for heavy Higgs searches in [75, 76]. There are also several theoretical studies showing the hypothesized sensitivity of the $14 \mathrm{TeV}$ LHC in the $m_{A}-\tan \beta$ plane due to different search channels, for example ref. [77]. In figures 4 and 5 we show the interplay between possible interesting signatures for $H / A$ searches at the LHC and the GCE best fit regions in the $m_{A}-\tan \beta$ plane, plotting contours of various branching ratios of interest for $H / A$ searches at the LHC. To highlight the regions of interest, we overlay the $\chi^{2}$ values from figure 1 as dashed blue lines for Fermi spectrum (b) in figure 4 and spectrum (d) in figure 5. The gray shaded regions denote the current LHC exclusion limits from searches for $H / A \rightarrow \tau^{+} \tau^{-}$at the $8 \mathrm{TeV}$ LHC (1- $\sigma$ and $2-\sigma$ as labeled). In both figures, the two panels in the top rows show the branching ratios of the CP-odd Higgs: $A \rightarrow \tau^{+} \tau^{-}$(left) and $A \rightarrow Z h$ (right). The lower four panels display the branching ratios for $H \rightarrow \tau^{+} \tau^{-}$(middle left), $H \rightarrow W^{+} W^{-}$(middle right), $H \rightarrow Z Z$ (lower left) and $H \rightarrow h h$ (lower right).

The top row shows that both $B R(A \rightarrow \tau \tau)$ and $B R(A \rightarrow Z h)$ are a few percent throughout the parameter region of interest, with the former always comparable to or larger (in some cases, by more than an order of magnitude). We can understand this behavior by noting that due to the close to alignment conditions, the $A Z h$ coupling is very suppressed. Hence, despite the $\tan \beta$ enhancement of the gluon fusion production of $A$, we find that the rates for $A \rightarrow Z h$ are at least 2 orders of magnitudes smaller than the current exclusion limits $[78,79]$ and therefore unlikely to be probed even at the high luminosity LHC $[75,76]$. Due to the absence of any other relevant decay modes, the decays to down-type fermions will still be the dominant decay modes and offer the best prospects for discovery of the pseudoscalar.

For the heavier CP-even Higgs $H$, in addition to the $\tau^{+} \tau^{-}$channel, there are nonnegligible branching ratios into $W W$ or $h h$ despite being suppressed due to alignment (recall that, close to alignment, $H \approx H_{N S M}$ ). These branching ratios are largest at low $\tan \beta$ below the top mass threshold, whereas $\operatorname{Br}\left(H \rightarrow \tau^{+} \tau^{-}\right)$is larger at higher $\tan \beta$. Note again that in the low $\tan \beta$ region, the main production of $H$ is via gluon fusion, which is enhanced due to the large unsuppressed top coupling. We computed the rate of $H \rightarrow W W$ relative to the SM expectation, $\mathcal{R}_{W W}^{H}$, which is shown as colored contours in figure 6. Current bounds on $\mathcal{R}_{W W}^{H}$ are at the level of $0.05-0.25$ [80], hence dedicated searches at the LHC could probe the GCE best-fit regions, particularly for $m_{A} \lesssim 350 \mathrm{GeV}$, where $\mathcal{R}_{W W}^{H}$ can be within a factor of 10 of the current exclusion limit $[75,76]$.

For $H / A$ heavier than about $350 \mathrm{GeV}$ and low values of $\tan \beta(\lesssim 7)$, both the CP-odd and even Higgs bosons preferentially decay to top quark pairs. However, due to the large 

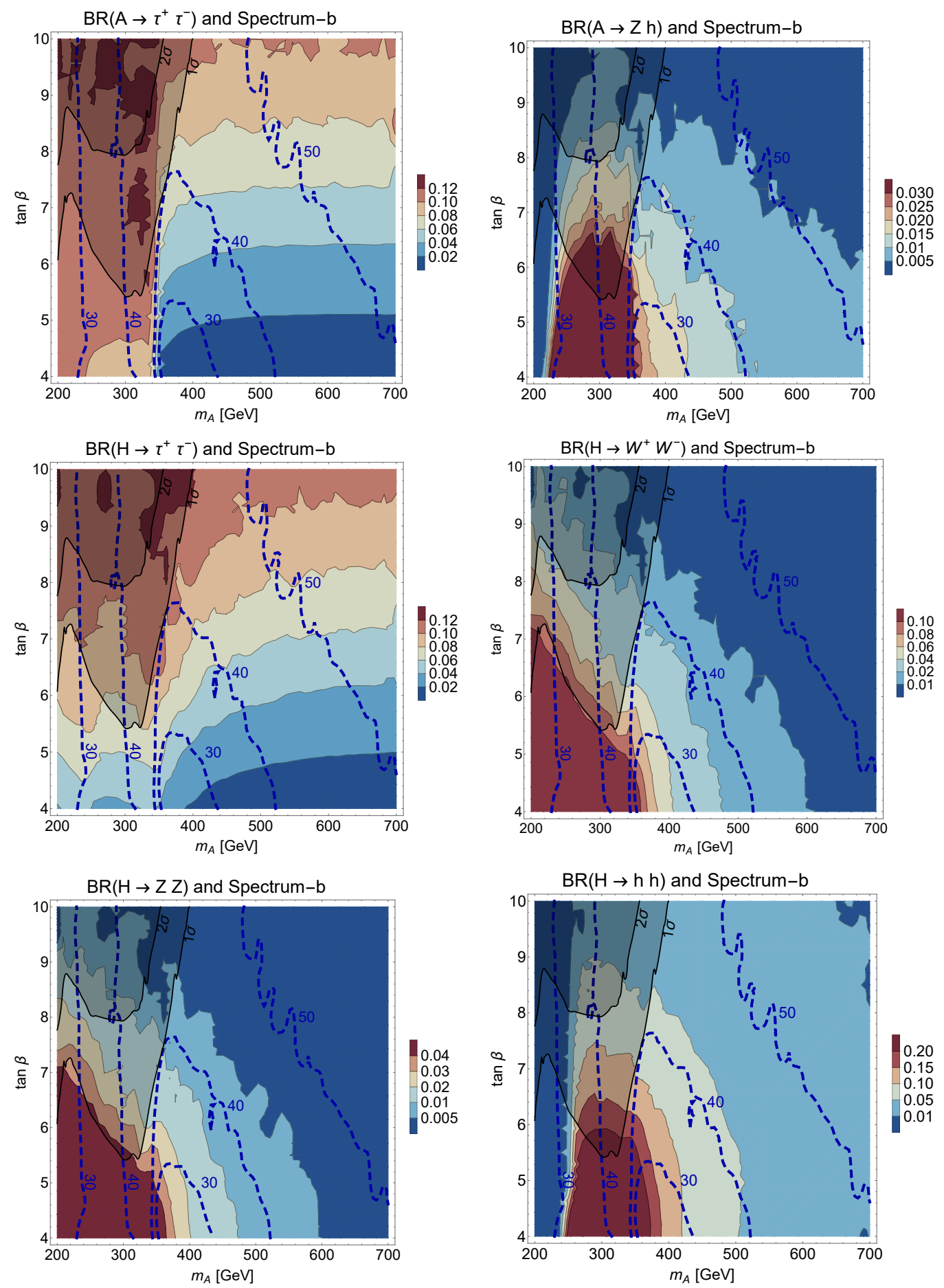

Figure 4. Predictions for LHC. Top: $B R\left(A \rightarrow \tau^{+} \tau^{-}\right)$and $B R(A \rightarrow Z h)$. Middle: $B R(H \rightarrow$ $\left.\tau^{+} \tau^{-}\right)$and $B R\left(H \rightarrow W^{+} W^{-}\right)$. Bottom: $B R(H \rightarrow Z Z)$ and $B R(H \rightarrow h h)$. Dashed blue lines show $\chi^{2}$ values from fitting the GCE to spectrum-b, as seen in figure 1 . The colored contour regions (and bar on the right) are each plot's respective branching ratio values. Shaded regions labelled 1- $\sigma$ and $2-\sigma$ are the $A / H \rightarrow \tau^{+} \tau^{-}$exclusion limits. 

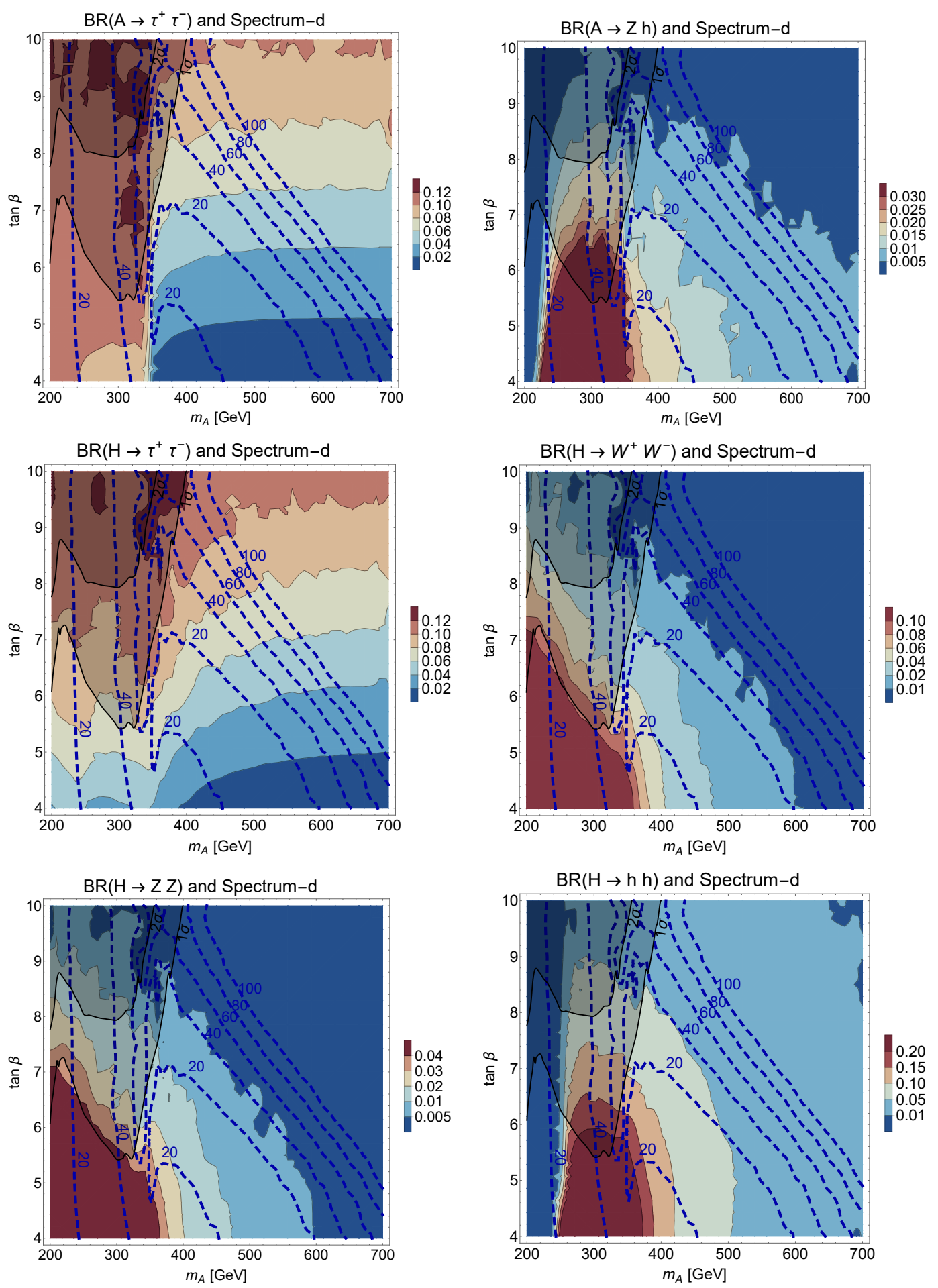

Figure 5. Same as figure 4 but with $\chi^{2}$ values from fitting the Galactic Center excess to spectrum$\mathrm{d}$, as shown in figure 1 . 


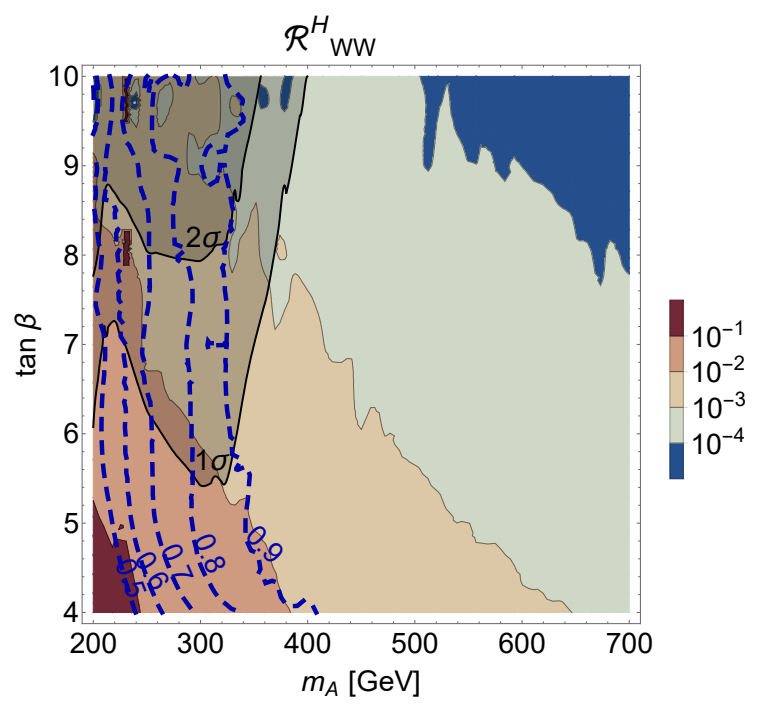

Figure 6. Shaded contours denote values of $\mathcal{R}_{W W}^{H}$. Gray shaded regions bounded by solid black lines show 1- $\sigma$ and 2- $\sigma$ exclusions by the $H / A \rightarrow \tau^{+} \tau^{-}$searches from the $8 \mathrm{TeV}$ LHC run (excluded above). The dashed blue lines correspond to contours of $\mathcal{R}_{W W}^{h}$.

SM $t \bar{t}$ background, this is a very challenging signature for the LHC [81, 82]; nevertheless, stronger sensitivity is expected at a $100 \mathrm{TeV}$ collider [82]. The standard $\tau^{+} \tau^{-}$searches can probe regions with larger values of $\tan \beta$.

It should be kept in mind that, in addition to these searches for heavier Higgs bosons, the good fit regions at low $m_{A} \lesssim 350 \mathrm{GeV}$ also predict deviations in $R_{W W}^{h}$ (see eq. (3.8) for definition) at the $10 \%$ level or more, hence such deviations from SM-like properties of the $125 \mathrm{GeV}$ Higgs could be a stark signal of this scenario. All of the above search modes as well as the precision measurements of the $125 \mathrm{GeV}$ Higgs are expected to improve substantially in sensitivity with the higher luminosity and energy of the $13 \mathrm{TeV}$ LHC [45, 46].

\subsection{Direct detection}

Our predictions for spin-independent direct detection experiments are plotted in figure 7, which shows DM masses and spin-independent DM-nucleon (proton) direct detection cross sections compatible with the GCE (Fermi spectrum (b) in blue, spectrum (d) in red). We only show points with $\chi^{2} \leq 50$ that are compatible with both the $2 \sigma A / H \rightarrow \tau^{+} \tau^{-}$ $8 \mathrm{TeV}$ LHC constraints and $0.7 \leq \mathcal{R}_{W W}^{h} \leq 1.3$. As discussed in section 3.3, we see that $\mathrm{DM}$ via the pseudoscalar resonance corresponds to generic cross sections of $\mathcal{O}\left(10^{-11}\right) \mathrm{pb}$, and these are comfortably safe from the existing Xenon100 [54] and LUX [55] bounds. A major fraction of the predicted parameter space can be probed with the next generation of direct detection experiments such as Xenon1T and LZ [83]. We note that almost all points predicted from our fit lie above the neutrino floor and therefore a signal can in principle be detected. The green cross and star correspond to the best fit points from figure 1 for spectrum (b) and (d) respectively. 


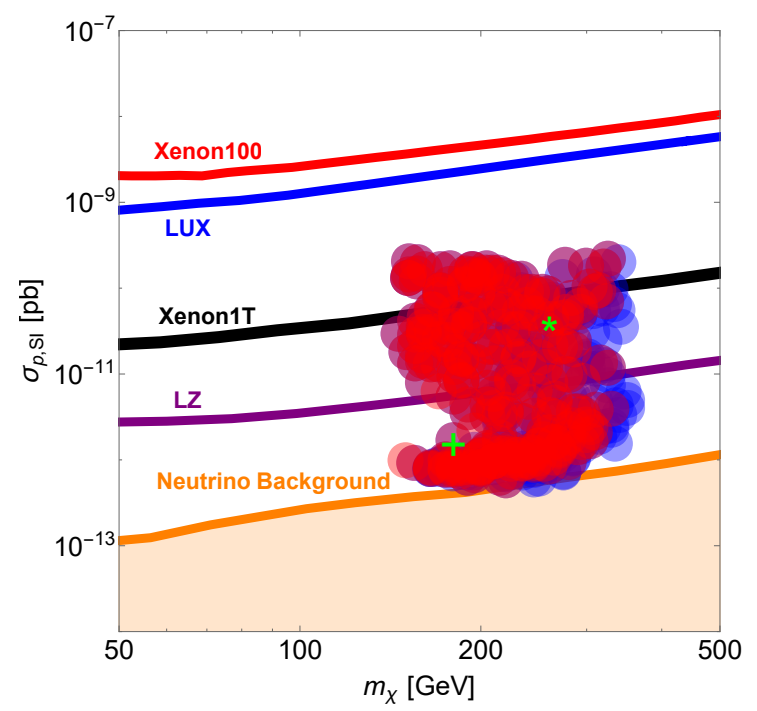

Figure 7. Dark matter masses, $m_{\chi}$, and spin-independent DM-nucleon (proton) direct detection cross sections, $\sigma_{S I}^{p}$, predicted by our fits to the Fermi GCE. Points compatible with Fermi spectrum b (d) are in blue (red); we have only plotted points with $\chi^{2} \leq 50$ and compatible with collider and Higgs data (see text). The green cross and star correspond to the best fit points for spectrum (b) and (d) respectively. Figure 1 shows $\chi^{2}$ contour regions from fitting the galactic center excess to Fermi spectrum (b) and (d). Current bounds (Xenon100, LUX), the reach of upcoming detectors (Xenon1T, LZ), and the neutrino background floor are also shown [83].

\section{Summary}

To conclude, we summarize the main findings of this paper:

- Recent reanalysis of GC background has found that the GCE could be consistent with annihilation of DM with much higher masses [15, 17, 18, 22]. This allows the GCE to be explained by the MSSM pseudoscalar resonance or " $A$-funnel". We fit to two different dark matter spectra, Fermi spectrum (b) and (d) from [15, 22], and find that reasonable fits can be obtained while maintaining consistency with stringent constraints from collider searches, Higgs data, and direct and indirect detection.

- For spectrum (b), the best fit region corresponds to $350 \mathrm{GeV} \lesssim m_{A} \lesssim 450 \mathrm{GeV}$ and $\tan \beta \lesssim 6$. This region can be probed with searches for $H \rightarrow W W$ and $t \bar{t}$ resonance searches. $m_{A} \lesssim 250 \mathrm{GeV}$ also gives reasonable fits but is incompatible with Higgs data.

- For spectrum (d), there are two regions with reasonable fits to the GCE: $450 \mathrm{GeV}$ $\lesssim m_{A} \lesssim 600 \mathrm{GeV}$ at $\tan \beta \lesssim 8$, and $m_{A} \sim 300$ and $\tan \beta \lesssim 5.5$. The former region can yield signals at the LHC in the $A / H \rightarrow \tau \tau$ or $t \bar{t}$ resonance searches at the LHC. The latter region can also be probed with the same channels, and should also lead to measurements of deviations of the $125 \mathrm{GeV}$ Higgs couplings from SM-like values. 
- The best fit regions for both spectra (b) and (d) predict spin-independent direct detection cross sections of $\mathcal{O}\left(10^{-11}\right)$ pb for a $110 \mathrm{GeV} \lesssim m_{\chi} \lesssim 350 \mathrm{GeV}$ neutralino. The entire region lies above the neutrino background, and the majority of the region is within reach of Xenon1T and LZ (see figure 7).

This exercise therefore leads to very sharp predictions for the next round of the LHC and direct detection experiments. Although the best fits obtained in this paper are noticeably worse than the best fit dark matter scenarios discussed elsewhere in literature, this highly predictive framework, coupled with the wide popularity of the MSSM, makes these results noteworthy. Even if the GCE turns out to be incompatible with the MSSM pseudoscalar resonance and is ultimately explained by some other (dark matter or astrophysical) phenomenon, this study still serves as a valuable template for the interplay between existing collider and Higgs constraints and the indirect, direct, and collider signatures of the $A$-funnel region with a light pseudoscalar in the MSSM.

\section{Acknowledgments}

It is our pleasure to thank Prateek Agrawal, Patrick Fox, Dan Hooper, Simona Murgia, Christopher Savage, and Carlos Wagner for valuable conversations. This work is supported by the U.S. Department of Energy, Office of Science, Office of High Energy Physics under Award Number DE-SC0007859. KF and AL are grateful for financial support from the Swedish Research Council (VR) through the Oskar Klein Centre and Stockholm University. AL is also supported by the Department of Physics at the University of Michigan, Ann Arbor. NS thanks the Aspen Center for Physics and the NSF Grant \#1066293 for hospitality during the completion of this work. NS is supported by a Wayne State University Research Grant Program award.

\section{A Parameters and vacuum metastability}

Figure 8 presents contour plots of the scanned parameters in the $m_{A}$ - $\tan \beta$ plane. The approximate check for vacuum metastability from eq. (3.10) is shown in figure 9 . It is seen that the desired condition is satisfied (corresponding to the plotted ratio being less than 1) in most of the parameter space not ruled out by the $8 \mathrm{TeV}$ LHC $A / H \rightarrow \tau^{+} \tau^{-}$bound.

Open Access. This article is distributed under the terms of the Creative Commons Attribution License (CC-BY 4.0), which permits any use, distribution and reproduction in any medium, provided the original author(s) and source are credited. 

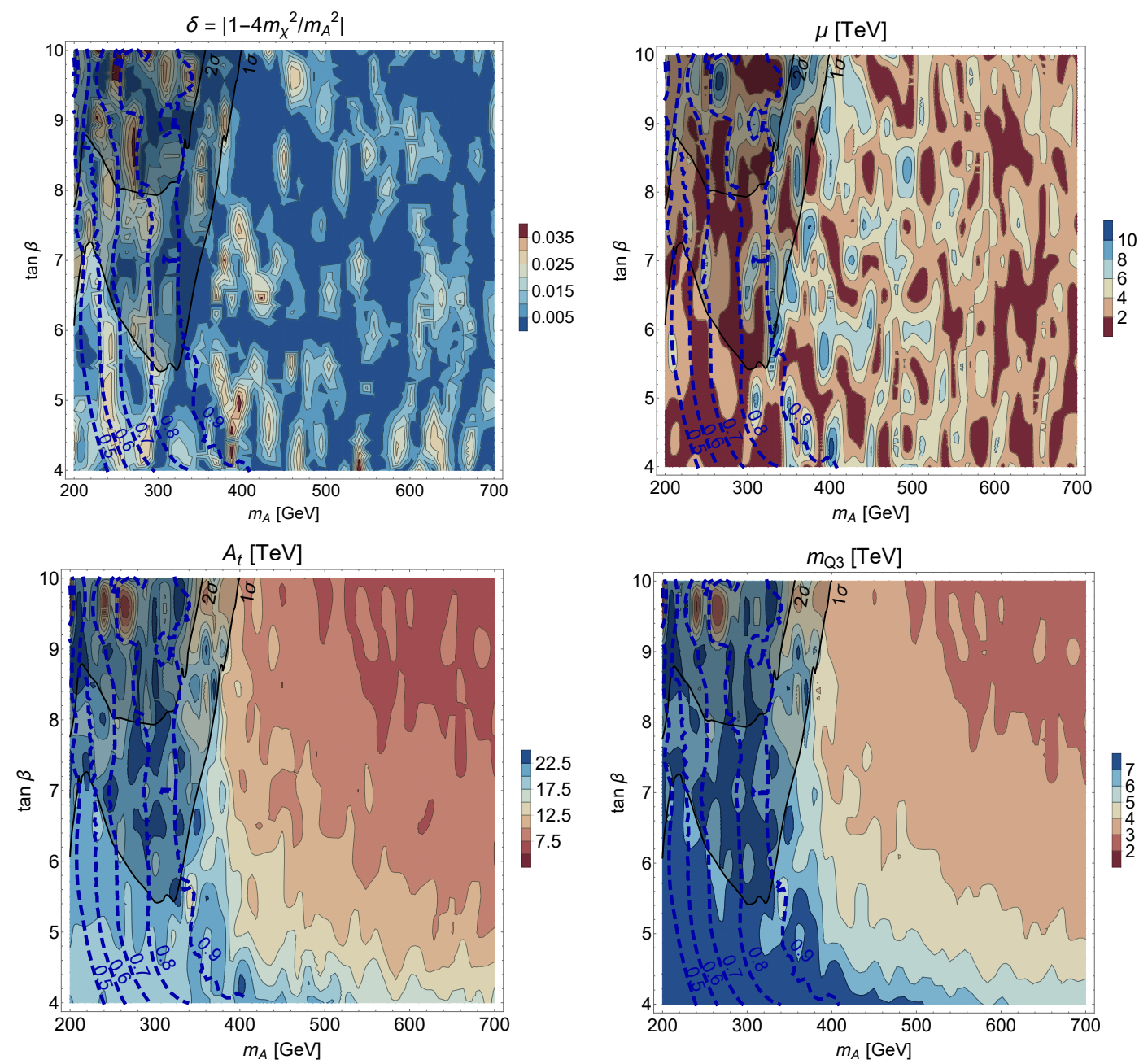

Figure 8. Contours of various input parameter values in the scan region. See text for details.

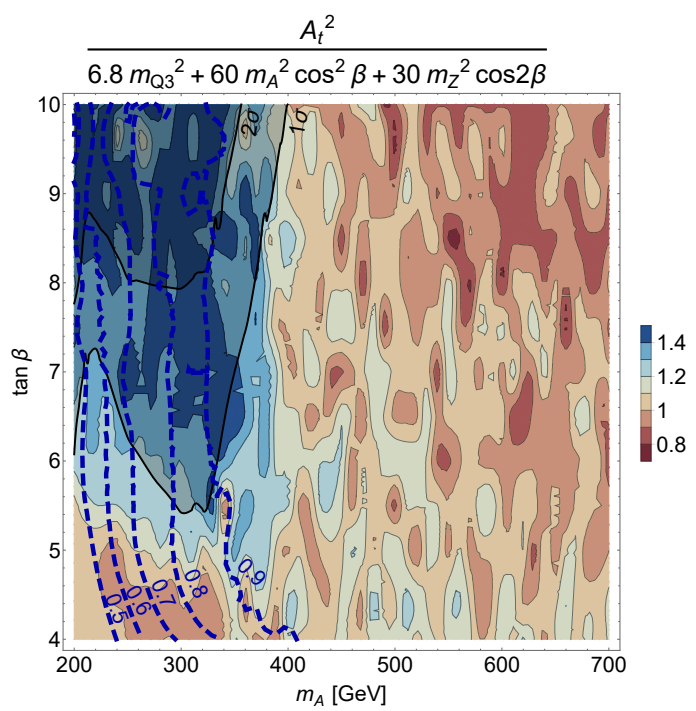

Figure 9. Vacuum metastability requires this ratio to be approximately less than 1 [47], so we see that most of our points are compatible with vacuum metastability bounds. 


\section{References}

[1] L. Goodenough and D. Hooper, Possible Evidence For Dark Matter Annihilation In The Inner Milky Way From The Fermi Gamma Ray Space Telescope, arXiv:0910.2998 [INSPIRE].

[2] D. Hooper and L. Goodenough, Dark Matter Annihilation in The Galactic Center As Seen by the Fermi Gamma Ray Space Telescope, Phys. Lett. B 697 (2011) 412 [arXiv:1010.2752] [INSPIRE].

[3] A. Boyarsky, D. Malyshev and O. Ruchayskiy, A comment on the emission from the Galactic Center as seen by the Fermi telescope, Phys. Lett. B 705 (2011) 165 [arXiv:1012.5839] [INSPIRE].

[4] D. Hooper and T. Linden, On The Origin Of The Gamma Rays From The Galactic Center, Phys. Rev. D 84 (2011) 123005 [arXiv:1110.0006] [INSPIRE].

[5] T. Linden, E. Lovegrove and S. Profumo, The Morphology of Hadronic Emission Models for the Gamma-Ray Source at the Galactic Center, Astrophys. J. 753 (2012) 41 [arXiv:1203.3539] [INSPIRE].

[6] K.N. Abazajian and M. Kaplinghat, Detection of a Gamma-Ray Source in the Galactic Center Consistent with Extended Emission from Dark Matter Annihilation and Concentrated Astrophysical Emission, Phys. Rev. D 86 (2012) 083511 [Erratum ibid. D 87 (2013) 129902] [arXiv:1207.6047] [INSPIRE].

[7] D. Hooper and T.R. Slatyer, Two Emission Mechanisms in the Fermi Bubbles: A Possible Signal of Annihilating Dark Matter, Phys. Dark Univ. 2 (2013) 118 [arXiv:1302.6589] [INSPIRE].

[8] C. Gordon and O. Macias, Dark Matter and Pulsar Model Constraints from Galactic Center Fermi-LAT Gamma Ray Observations, Phys. Rev. D 88 (2013) 083521 [arXiv:1306.5725] [INSPIRE].

[9] K.N. Abazajian, N. Canac, S. Horiuchi and M. Kaplinghat, Astrophysical and Dark Matter Interpretations of Extended Gamma-Ray Emission from the Galactic Center, Phys. Rev. D 90 (2014) 023526 [arXiv: 1402.4090] [INSPIRE].

[10] T. Daylan et al., The characterization of the gamma-ray signal from the central Milky Way: A case for annihilating dark matter, Phys. Dark Univ. 12 (2016) 1 [arXiv:1402.6703] [INSPIRE].

[11] B. Zhou et al., GeV excess in the Milky Way: The role of diffuse galactic gamma-ray emission templates, Phys. Rev. D 91 (2015) 123010 [arXiv:1406.6948] [INSPIRE].

[12] F. Calore, I. Cholis and C. Weniger, Background model systematics for the Fermi GeV excess, JCAP 03 (2015) 038 [arXiv: 1409.0042] [INSPIRE].

[13] F. Calore, I. Cholis, C. McCabe and C. Weniger, A Tale of Tails: Dark Matter Interpretations of the Fermi GeV Excess in Light of Background Model Systematics, Phys. Rev. D 91 (2015) 063003 [arXiv:1411.4647] [InSPIRE].

[14] M. Abdullah, A. DiFranzo, A. Rajaraman, T.M.P. Tait, P. Tanedo and A.M. Wijangco, Hidden on-shell mediators for the Galactic Center $\gamma$-ray excess, Phys. Rev. D 90 (2014) 035004 [arXiv: 1404.6528] [INSPIRE].

[15] P. Agrawal, B. Batell, P.J. Fox and R. Harnik, WIMPs at the Galactic Center, JCAP 05 (2015) 011 [arXiv: 1411.2592] [INSPIRE]. 
[16] M. Cahill-Rowley, J. Gainer, J. Hewett and T. Rizzo, Towards a Supersymmetric Description of the Fermi Galactic Center Excess, JHEP 02 (2015) 057 [arXiv: 1409.1573] [INSPIRE].

[17] A. Achterberg, S. Amoroso, S. Caron, L. Hendriks, R. Ruiz de Austri and C. Weniger, A description of the Galactic Center excess in the Minimal Supersymmetric Standard Model, JCAP 08 (2015) 006 [arXiv: 1502.05703] [INSPIRE].

[18] G. Bertone et al., Global analysis of the pMSSM in light of the Fermi GeV excess: prospects for the LHC Run-II and astroparticle experiments, arXiv:1507.07008 [INSPIRE].

[19] S.K. Lee, M. Lisanti, B.R. Safdi, T.R. Slatyer and W. Xue, Evidence for Unresolved $\gamma$-Ray Point Sources in the Inner Galaxy, Phys. Rev. Lett. 116 (2016) 051103 [arXiv:1506.05124] [INSPIRE].

[20] R. Bartels, S. Krishnamurthy and C. Weniger, Strong support for the millisecond pulsar origin of the Galactic center GeV excess, Phys. Rev. Lett. 116 (2016) 051102 [arXiv: 1506. 05104] [INSPIRE].

[21] D. Gaggero, M. Taoso, A. Urbano, M. Valli and P. Ullio, Towards a realistic astrophysical interpretation of the gamma-ray Galactic center excess, JCAP 12 (2015) 056 [arXiv: 1507.06129] [INSPIRE].

[22] Fermi-LAT collaboration, S. Murgia, Observation of the High Energy Gamma-ray Emission Towards the Galactic Center, http://fermi.gsfc.nasa.gov/science/mtgs/symposia/2014/program/08_Murgia.pdf (2014).

[23] FERmi-LAT collaboration, M. Ajello et al., Fermi-LAT Observations of High-Energy $\gamma$-Ray Emission Toward the Galactic Center, Astrophys. J. 819 (2016) 44 [arXiv:1511.02938] [INSPIRE].

[24] A. Berlin, S. Gori, T. Lin and L.-T. Wang, Pseudoscalar Portal Dark Matter, Phys. Rev. D 92 (2015) 015005 [arXiv: 1502.06000] [INSPIRE].

[25] C. Cheung, M. Papucci, D. Sanford, N.R. Shah and K.M. Zurek, NMSSM Interpretation of the Galactic Center Excess, Phys. Rev. D 90 (2014) 075011 [arXiv:1406.6372] [InSPIRE].

[26] S. Ipek, D. McKeen and A.E. Nelson, A Renormalizable Model for the Galactic Center Gamma Ray Excess from Dark Matter Annihilation, Phys. Rev. D 90 (2014) 055021 [arXiv: 1404.3716] [INSPIRE].

[27] ATLAS collaboration, Search for charged Higgs bosons in the $\tau+$ jets final state with $p p$ collision data recorded at $\sqrt{s}=8 \mathrm{TeV}$ with the ATLAS experiment, ATLAS-CONF-2013-090 (2013).

[28] CMS collaboration, Search for a light charged Higgs boson in top quark decays in pp collisions at $\sqrt{s}=7 \mathrm{TeV}$, JHEP 07 (2012) 143 [arXiv:1205.5736] [INSPIRE].

[29] A. Berlin, P. Gratia, D. Hooper and S.D. McDermott, Hidden Sector Dark Matter Models for the Galactic Center Gamma-Ray Excess, Phys. Rev. D 90 (2014) 015032 [arXiv:1405.5204] [INSPIRE].

[30] M. Carena, I. Low, N.R. Shah and C.E.M. Wagner, Impersonating the Standard Model Higgs Boson: Alignment without Decoupling, JHEP 04 (2014) 015 [arXiv:1310.2248] [INSPIRE].

[31] M. Carena, H.E. Haber, I. Low, N.R. Shah and C.E.M. Wagner, Complementarity between Nonstandard Higgs Boson Searches and Precision Higgs Boson Measurements in the MSSM, Phys. Rev. D 91 (2015) 035003 [arXiv:1410.4969] [inSPIRE].

[32] J.F. Gunion and H.E. Haber, The CP conserving two Higgs doublet model: The Approach to the decoupling limit, Phys. Rev. D 67 (2003) 075019 [hep-ph/0207010] [INSPIRE]. 
[33] D.M. Asner et al., ILC Higgs White Paper, in Community Summer Study 2013 : Snowmass on the Mississippi. (CSS2013), Minneapolis U.S.A. (2013) [arXiv:1310.0763] [INSPIRE].

[34] H.E. Haber, The Higgs data and the Decoupling Limit, in 1st Toyama International Workshop on Higgs as a Probe of New Physics 2013 (HPNP2013), Toyama Japan (2013) [arXiv: 1401.0152] [INSPIRE].

[35] N. Craig, J. Galloway and S. Thomas, Searching for Signs of the Second Higgs Doublet, arXiv:1305.2424 [INSPIRE].

[36] M. Perelstein and B. Shakya, Fine-Tuning Implications of Direct Dark Matter Searches in the MSSM, JHEP 10 (2011) 142 [arXiv:1107.5048] [INSPIRE].

[37] S. Amsel, K. Freese and P. Sandick, Probing EWSB Naturalness in Unified SUSY Models with Dark Matter, JHEP 11 (2011) 110 [arXiv:1108.0448] [INSPIRE].

[38] M. Perelstein and B. Shakya, XENON100 implications for naturalness in the MSSM, NMSSM and $\lambda$-supersymmetry model, Phys. Rev. D 88 (2013) 075003 [arXiv:1208.0833] [INSPIRE].

[39] MSSM Working Group collaboration, A. Djouadi et al., The Minimal supersymmetric standard model: Group summary report, hep-ph/9901246 [INSPIRE].

[40] K. Griest and D. Seckel, Three exceptions in the calculation of relic abundances, Phys. Rev. D 43 (1991) 3191.

[41] CMS collaboration, Search for neutral MSSM Higgs bosons decaying to a pair of tau leptons in pp collisions, JHEP 10 (2014) 160 [arXiv:1408.3316] [INSPIRE].

[42] W. Altmannshofer, M. Carena, N.R. Shah and F. Yu, Indirect Probes of the MSSM after the Higgs Discovery, JHEP 01 (2013) 160 [arXiv:1211.1976] [INSPIRE].

[43] ATLAS collaboration, Measurements of the Higgs boson production and decay rates and coupling strengths using pp collision data at $\sqrt{s}=7$ and $8 \mathrm{TeV}$ in the ATLAS experiment, Eur. Phys. J. C 76 (2016) 6 [arXiv: 1507.04548] [InSPIRE].

[44] CMS collaboration, Precise determination of the mass of the Higgs boson and tests of compatibility of its couplings with the standard model predictions using proton collisions at 7 and $8 \mathrm{TeV}$, Eur. Phys. J. C 75 (2015) 212 [arXiv:1412.8662] [INSPIRE].

[45] ATLAS collaboration, Projections for measurements of Higgs boson signal strengths and coupling parameters with the ATLAS detector at a HL-LHC, ATL-PHYS-PUB-2014-016 (2014).

[46] CMS collaboration, Projected Performance of an Upgraded CMS Detector at the LHC and HL-LHC: Contribution to the Snowmass Process, in Community Summer Study 2013 : Snowmass on the Mississippi. (CSS2013), Minneapolis U.S.A. (2013) [arXiv:1307.7135] [INSPIRE].

[47] N. Blinov and D.E. Morrissey, Vacuum Stability and the MSSM Higgs Mass, JHEP 03 (2014) 106 [arXiv:1310.4174] [InSPIRE].

[48] A. Anandakrishnan, B. Shakya and K. Sinha, Dark matter at the pseudoscalar Higgs resonance in the phenomenological MSSM and SUSY GUTs, Phys. Rev. D 91 (2015) 035029 [arXiv: 1410.0356] [INSPIRE].

[49] D. Hooper, C. Kelso, P. Sandick and W. Xue, Closing Supersymmetric Resonance Regions With Direct Detection Experiments, Phys. Rev. D 88 (2013) 015010 [arXiv:1304.2417] [INSPIRE]. 
[50] T. Han, Z. Liu and A. Natarajan, Dark matter and Higgs bosons in the MSSM, JHEP 11 (2013) 008 [arXiv: 1303.3040] [INSPIRE].

[51] J. Hisano, K. Ishiwata and N. Nagata, Gluon contribution to the dark matter direct detection, Phys. Rev. D 82 (2010) 115007 [arXiv: 1007.2601] [INSPIRE].

[52] C. Cheung, L.J. Hall, D. Pinner and J.T. Ruderman, Prospects and Blind Spots for Neutralino Dark Matter, JHEP 05 (2013) 100 [arXiv:1211.4873] [INSPIRE].

[53] Y.G. Kim, T. Nihei, L. Roszkowski and R. Ruiz de Austri, Upper and lower limits on neutralino WIMP mass and spin independent scattering cross-section and impact of new (g-2)(mu) measurement, JHEP 12 (2002) 034 [hep-ph/0208069] [INSPIRE].

[54] XENON100 collaboration, E. Aprile et al., Limits on spin-dependent WIMP-nucleon cross sections from 225 live days of XENON100 data, Phys. Rev. Lett. 111 (2013) 021301 [arXiv: 1301.6620] [INSPIRE].

[55] LUX collaboration, D.S. Akerib et al., First results from the LUX dark matter experiment at the Sanford Underground Research Facility, Phys. Rev. Lett. 112 (2014) 091303 [arXiv:1310.8214] [INSPIRE].

[56] Fermi-LAT collaboration, M. Ackermann et al., Searching for Dark Matter Annihilation from Milky Way Dwarf Spheroidal Galaxies with Six Years of Fermi Large Area Telescope Data, Phys. Rev. Lett. 115 (2015) 231301 [arXiv: 1503.02641] [INSPIRE].

[57] A. Geringer-Sameth et al., Indication of Gamma-ray Emission from the Newly Discovered Dwarf Galaxy Reticulum II, Phys. Rev. Lett. 115 (2015) 081101 [arXiv:1503.02320] [INSPIRE].

[58] DES collaboration, K. Bechtol et al., Eight New Milky Way Companions Discovered in First-Year Dark Energy Survey Data, Astrophys. J. 807 (2015) 50 [arXiv:1503.02584] [INSPIRE].

[59] DES, Fermi-LAT collaboration, A. Drlica-Wagner et al., Search for Gamma-Ray Emission from DES Dwarf Spheroidal Galaxy Candidates with Fermi-LAT Data, Astrophys. J. 809 (2015) L4 [arXiv: 1503.02632] [INSPIRE].

[60] Planck collaboration, P.A.R. Ade et al., Planck 2015 results. XIII. Cosmological parameters, arXiv: 1502.01589 [INSPIRE].

[61] K. Kong and J.-C. Park, Bounds on dark matter interpretation of Fermi-LAT GeV excess, Nucl. Phys. B 888 (2014) 154 [arXiv:1404.3741] [InSPIRE].

[62] T. Bringmann, M. Vollmann and C. Weniger, Updated cosmic-ray and radio constraints on light dark matter: Implications for the GeV gamma-ray excess at the Galactic center, Phys. Rev. D 90 (2014) 123001 [arXiv:1406.6027] [InSPIRE].

[63] M. Cirelli, D. Gaggero, G. Giesen, M. Taoso and A. Urbano, Antiproton constraints on the GeV gamma-ray excess: a comprehensive analysis, JCAP 12 (2014) 045 [arXiv:1407.2173] [INSPIRE].

[64] M. Cirelli and G. Giesen, Antiprotons from Dark Matter: Current constraints and future sensitivities, JCAP 04 (2013) 015 [arXiv:1301.7079] [INSPIRE].

[65] M. Perelstein and B. Shakya, Antiprotons from Dark Matter: Effects of a Position-Dependent Diffusion Coefficient, Phys. Rev. D 83 (2011) 123508 [arXiv: 1012.3772] [INSPIRE].

[66] N. Fornengo, L. Maccione and A. Vittino, Constraints on particle dark matter from cosmic-ray antiprotons, JCAP 04 (2014) 003 [arXiv: 1312.3579] [INSPIRE]. 
[67] IceCube collaboration, M.G. Aartsen et al., Search for Dark Matter Annihilation in the Galactic Center with IceCube-79, Eur. Phys. J. C 75 (2015) 492 [arXiv:1505.07259] [INSPIRE].

[68] G. Bélanger, F. Boudjema, A. Pukhov and A. Semenov, MicrOMEGAs4.1: two dark matter candidates, Comput. Phys. Commun. 192 (2015) 322 [arXiv: 1407.6129] [INSPIRE].

[69] A. Djouadi, J.-L. Kneur and G. Moultaka, SuSpect: A Fortran code for the supersymmetric and Higgs particle spectrum in the MSSM, Comput. Phys. Commun. 176 (2007) 426 [hep-ph/0211331] [INSPIRE].

[70] T. Hahn, S. Heinemeyer, W. Hollik, H. Rzehak and G. Weiglein, High-Precision Predictions for the Light CP -Even Higgs Boson Mass of the Minimal Supersymmetric Standard Model, Phys. Rev. Lett. 112 (2014) 141801 [arXiv:1312.4937] [INSPIRE].

[71] M. Frank, T. Hahn, S. Heinemeyer, W. Hollik, H. Rzehak and G. Weiglein, The Higgs Boson Masses and Mixings of the Complex MSSM in the Feynman-Diagrammatic Approach, JHEP 02 (2007) 047 [hep-ph/0611326] [INSPIRE].

[72] G. Degrassi, S. Heinemeyer, W. Hollik, P. Slavich and G. Weiglein, Towards high precision predictions for the MSSM Higgs sector, Eur. Phys. J. C 28 (2003) 133 [hep-ph/0212020] [INSPIRE].

[73] S. Heinemeyer, W. Hollik and G. Weiglein, The Masses of the neutral CP - even Higgs bosons in the MSSM: Accurate analysis at the two loop level, Eur. Phys. J. C 9 (1999) 343 [hep-ph/9812472] [INSPIRE].

[74] S. Heinemeyer, W. Hollik and G. Weiglein, FeynHiggs: A Program for the calculation of the masses of the neutral CP even Higgs bosons in the MSSM, Comput. Phys. Commun. 124 (2000) 76 [hep-ph/9812320] [INSPIRE].

[75] ATLAS collaboration, Beyond-the-Standard-Model Higgs boson searches at a High-Luminosity LHC with ATLAS, ATLAS-PHYS-PUB-2013-016 (2013).

[76] CMS collaboration, 2HDM Neutral Higgs Future Analysis Studies, CMS-PAS-FTR-13-024 (2013).

[77] A. Djouadi, L. Maiani, A. Polosa, J. Quevillon and V. Riquer, Fully covering the MSSM Higgs sector at the LHC, JHEP 06 (2015) 168 [arXiv: 1502.05653] [INSPIRE].

[78] ATLAS collaboration, Search for a CP-odd Higgs boson decaying to Zh in pp collisions at $\sqrt{s}=8 \mathrm{TeV}$ with the ATLAS detector, Phys. Lett. B 744 (2015) 163 [arXiv:1502.04478] [INSPIRE].

[79] CMS collaboration, Search for a pseudoscalar boson decaying into a $Z$ boson and the $125 \mathrm{GeV}$ Higgs boson in $\ell^{+} \ell^{-} b \bar{b}$ final states, Phys. Lett. B 748 (2015) 221 [arXiv: 1504.04710] [INSPIRE].

[80] CMS collaboration, M. Pelliccioni, CMS High mass $W W$ and ZZ Higgs search with the complete LHC Run1 statistics, arXiv: 1505.03831 [INSPIRE].

[81] N. Craig, F. D'Eramo, P. Draper, S. Thomas and H. Zhang, The Hunt for the Rest of the Higgs Bosons, JHEP 06 (2015) 137 [arXiv: 1504.04630] [INSPIRE].

[82] J. Hajer, Y.-Y. Li, T. Liu and J.F.H. Shiu, Heavy Higgs Bosons at $14 \mathrm{TeV}$ and $100 \mathrm{TeV}$, JHEP 11 (2015) 124 [arXiv: 1504.07617] [INSPIRE].

[83] J.L. Feng et al., Planning the Future of U.S. Particle Physics (Snowmass 2013): Chapter 4: Cosmic Frontier, arXiv:1401.6085 [INSPIRE]. 